\title{
Transcytosis route mediates rapid delivery of intact antibodies to draining lymph nodes
}

\author{
Laura Kähäri, ${ }^{1,2}$ Ruth Fair-Mäkelä, ${ }^{1,2}$ Kaisa Auvinen,, ${ }^{1,2}$ Pia Rantakari,, ${ }^{1,2}$ Sirpa Jalkanen, ${ }^{1,2}$ Johanna Ivaska, ${ }^{3}$ and Marko Salmi' ${ }^{1,2}$ \\ ${ }^{1}$ MediCity Research Laboratory, University of Turku, Turku, Finland. ${ }^{2}$ Institute of Biomedicine, University of Turku, Turku, Finland. ${ }^{3}$ Centre of Biotechnology, University of Turku, Turku, Finland.
}

\begin{abstract}
Lymph nodes (LNs) filter lymph to mount effective immune responses. Small soluble lymph-borne molecules from the periphery enter the draining LNs via a reticular conduit system. Intact antibodies and other larger molecules, in contrast, are physically unable to enter the conduits, and they are thought to be transported to the LNs only within migratory DCs after proteolytic degradation. Here, we discovered that lymph-borne antibodies and other large biomolecules enter within seconds into the parenchyma of the draining LN in an intact form. Mechanistically, we found that the uptake of large molecules is a receptor-independent, fluid-phase process that takes place by dynamin-dependent vesicular transcytosis through the lymphatic endothelial cells in the subcapsular sinus of the LN. Physiologically, this pathway mediates a very fast transfer of large protein antigens from the periphery to LN-resident DCs and macrophages. We show that exploitation of the transcytosis system allows enhanced whole-organ imaging and spatially controlled lymphocyte activation by s.c. administered antibodies in vivo. Transcytosis through the floor of the subcapsular sinus thus represents what we believe to be a new physiological and targetable mode of lymph filtering.
\end{abstract}

\section{Introduction}

The lymphatic vasculature controls the clearance of fluid and molecules from all tissues (1-3). Endogenous molecules from the periphery as well as exogenous substances penetrating the epithelial barriers readily gain access to the afferent lymphatic vasculature. Soluble molecules including antigens, antibodies, chemokines, and other signaling molecules carried in the interstitial fluid penetrate the extracellular matrix and the button-like endothelial junctions of the terminal lymphatic capillaries relatively freely and nonselectively $(4,5)$. They then rapidly drain via the afferent lymphatics to the subcapsular sinus of the lymph node (LN).

The subcapsular sinus of the $\mathrm{LN}$ is rich in scavenging receptor- and Fc receptor-expressing macrophages, which capture and destroy many lymph-borne molecules (6). Certain small lymphborne molecules (<70 kDa in molecular mass), on the other hand, can pass through the floor of the subcapsular sinus by traversing narrow transendothelial channels covered with filter-like diaphragms. These molecules then enter adjacent reticular conduits, which distribute them within this cable-like network throughout the LN (7-11). In contrast, large (>70 kDa) soluble lymph-borne molecules, such as antibodies, cannot enter the reticular conduit system because of physical constraints imposed by the dimensions of the diaphragms guarding the conduit openings and by the spacing of collagen fibers at the core of the reticular conduits $(7$, $9,11,12)$. According to the prevailing concept, large protein antigens can only gain access to LNs in approximately 10 hours within

Authorship note: LK, RFM, and KA contributed equally to this work.

Conflict of interest: The authors have declared that no conflict of interest exists. Copyright: (5) 2019, American Society for Clinical Investigation.

Submitted: October 22, 2018; Accepted: May 10, 2019; Published: June 24, 2019.

Reference information: J Clin Invest. 2019;129(8):3086-3102.

https://doi.org/10.1172/JCl125740. migratory DCs after proteolytic degradation into small peptides, whereas other types of large biomolecules have no entrance into the LNs $(1,2,7-11)$.

When studying the function of the subcapsular sinus in LNs, we unexpectedly observed that intact lymph-borne $\operatorname{IgG}$ antibodies had instant access to the parenchyma of draining LNs in a process independent of reticular conduits. Since antibodies are physiological lymph-borne proteins $(13,14)$ and offer a convenient way of visualizing protein transport in vivo, we used them to dissect the transfer of large lymph-borne proteins through the sinus floor. Mechanistically, we show that the transfer takes place through subcapsular sinus lymphatic endothelial cells (LECs) in the fluid phase via an ultrafast dynamin-dependent transport system. We observed that other exogenous large protein and oligosaccharide antigens were also rapidly transferred to the parenchyma of draining LNs via the same mechanism. This allows the uptake of large antigens in resident LN antigen-presenting cells well before the arrival of the first migratory DCs. We demonstrate that the delivery of intact s.c. injected antibodies to the draining $\mathrm{LN}$ via this new transcytosis route can be harnessed to improve the speed ( $>100$-fold) and flexibility of whole-mount bioimaging. Therapeutically, we show that the transsinusoidal transfer mechanism allows site-selective, antibody-mediated activation of lymphocytes in a given $\mathrm{LN}$ in vivo. We believe these data profoundly alter our knowledge of LN functions and the pharmacokinetics of antibodies.

\section{Results}

Rapid transfer of lymph-borne antibodies to the parenchyma of the draining LNs. We originally observed that a lymph-borne antiendothelial antibody specifically stained not only the luminal surface of LECs in the subcapsular sinus of the draining LN but also blood vessel ECs (BECs) deep in the node parenchyma (Supplemental Figure 1A; supplemental material available online with this 
A

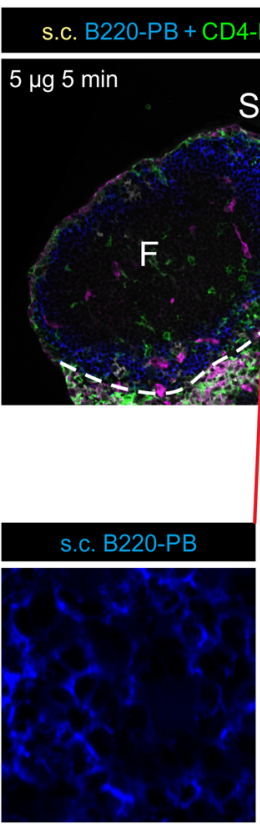

C

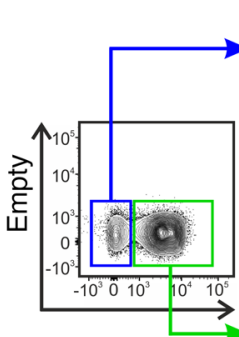

Ex vivo CD3-A647
Draining

FITC + CD11C-A647 + CD31-A594
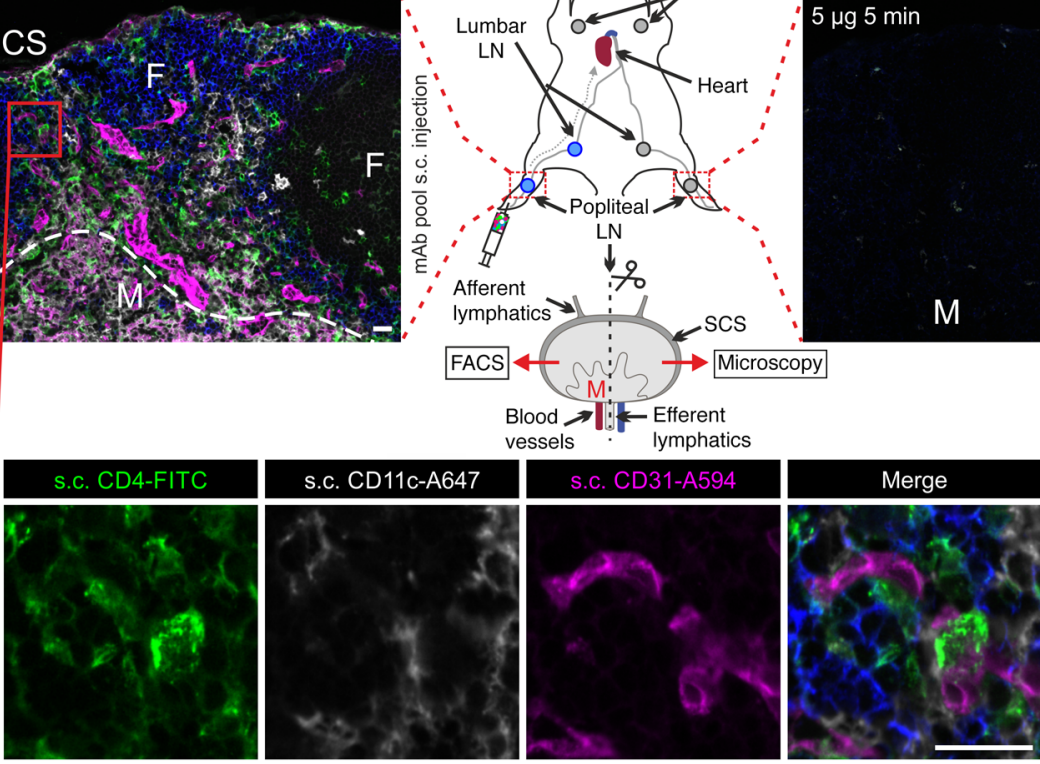

Nondraining s.c. B220-PB + CD4-FITC + CD11C-A647+CD31-A594

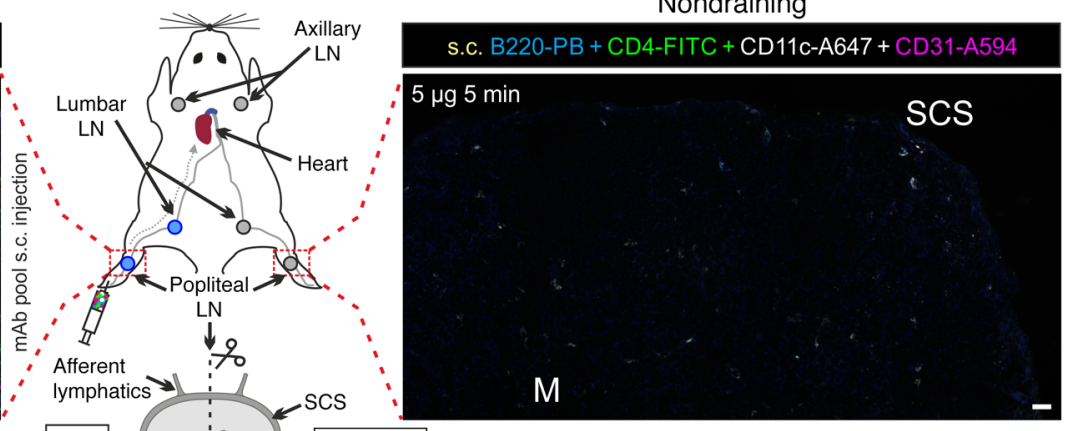

B

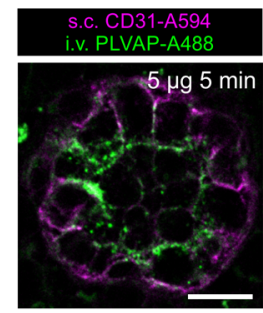

D

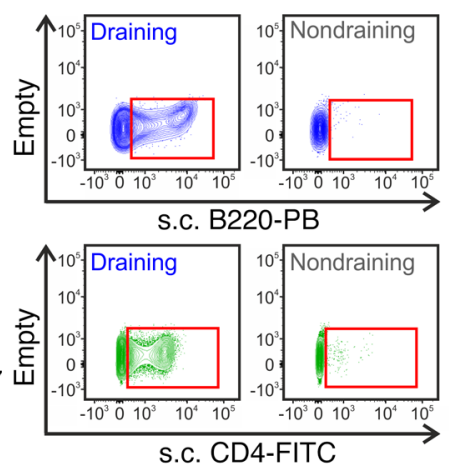

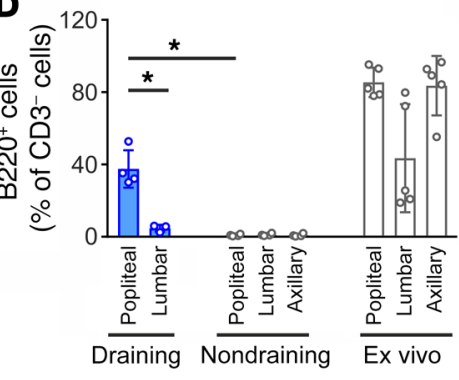

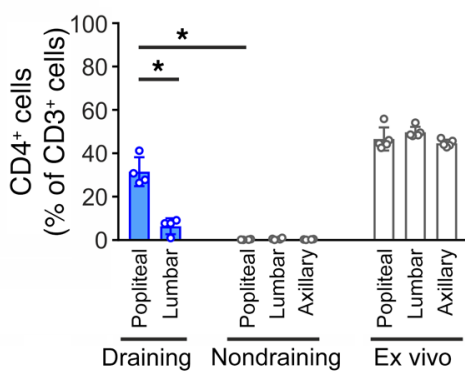

Figure 1. Transfer of lymph-borne antibodies to the parenchyma of the draining LNs. (A) Experimental setup and confocal analyses of the draining and nondraining popliteal LNs after s.C. administration of fluorochrome-conjugated monoclonal antibodies (Pacific Blue-conjugated anti-B220 [B220-PB],

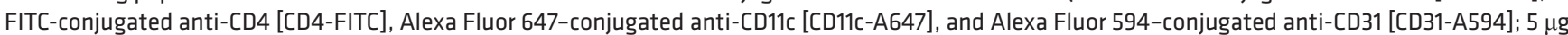
each, $t=5 \mathrm{~min}, n=4)$. SCS, subcapsular sinus; F, follicle; $M$, medulla. Scale bars: $20 \mu \mathrm{m}$. (B) Confocal analyses of a high endothelial venule in the draining LN after s.c. administration of Alexa Fluor 594-CD31 antibody ( $5-\mu \mathrm{g}$ dose, $t=5 \mathrm{~min}, n=4)$. The luminal surfaces of vessels were labeled by i.v. administration of Alexa Fluor 488-PLVAP antibody. Scale bar: $10 \mu \mathrm{m}$. (C and D) Flow cytometric analyses of lymphocytes in LNs after s.c. administration of fluorochrome-conjugated B220 and CD4 antibodies $(n=3-5)$. The cells were stained ex vivo for CD3. (C) Representative flow cytometric plots and the gating strategy. (D) Quantification of the antibody transfer to the draining (ipsilateral popliteal and lumbar) and nondraining (contralateral popliteal, lumbar, and axillary) LNs. Lymphocytes from untouched mice were stained ex vivo for B220, CD4, and CD3. In bar graphs, each dot represents 1 LN, and data are the mean $\pm S D$. ${ }^{*} P<0.05$, by Mann-Whitney $U$ test.

article; https://doi.org/10.1172/JCI125740DS1). We then administered 4 differently tagged monoclonal antibodies (Pacific Blueconjugated anti-B220 to detect B lymphocytes; FITC-conjugated anti-CD4 to detect Th lymphocytes; Alexa Fluor 647-conjugated anti-CD11c to detect DCs, and Alexa Fluor 549-conjugated antiCD31 to detect ECs) into the hind paws of WT mice (Figure 1A). When the draining popliteal LN was analyzed by microscopy 5 minutes later, we found that all 4 in vivo-administered antibodies had entered the parenchyma and specifically stained their intranodal target cell types in the cortex and paracortex (Figure 1A). In addition, all antibodies accumulated nonselectively in the LN medulla, which is known to harbor intraluminal medullary sinus macrophages active in scavenging many types of lymph-borne molecules (ref. 6; and therefore we did not study the medullary antibody uptake further). When we focused on the cortex, we observed that the lymph-borne antibodies penetrated both the extrafollicular areas and the B cell follicles (Figure 1A). The antibodies apparently penetrated the follicles more slowly than they did other areas of the cortex. We found that the lymph-borne CD31 antibody rapidly reached the EC plasma membrane in the parenchymal blood vessels in the cortex and paracortex (Figure 1B). Ex vivo overlay stainings with a different CD31 antibody confirmed that the binding of the lymph-borne anti-CD31 antibody was specific (Supplemental Figure 1B). Flow cytometric quantifications showed that the lymph-borne B220 and CD4 antibodies had stained a substantial portion of the total B and T cells, respec- 

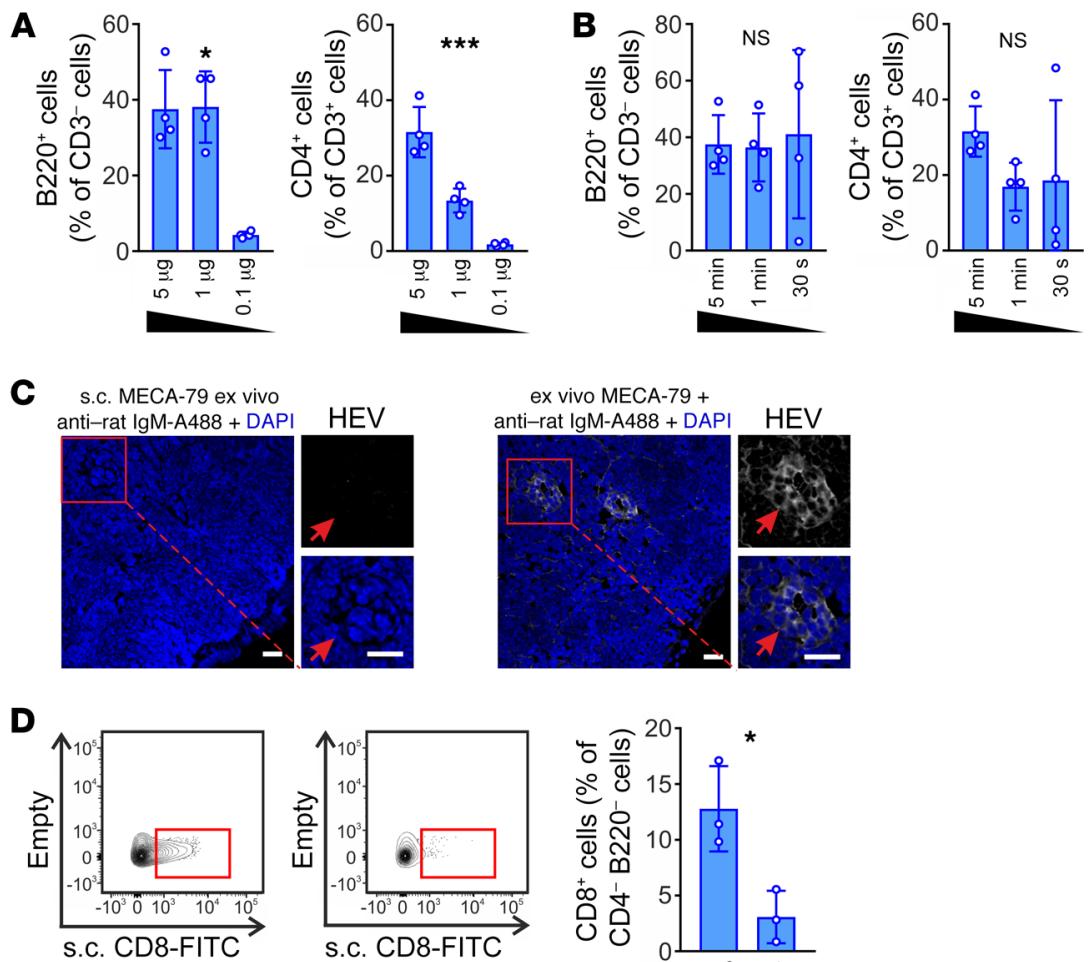

rat IgG2a
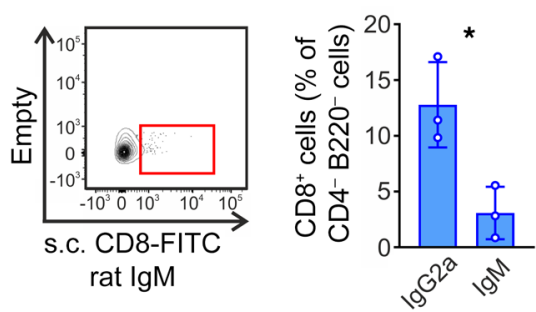

tively, in the draining LN (Figure 1, C and D). Notably, the more central draining LNs (lumbar nodes; i.e., those along the LN chain further away from the s.c. injection site) showed only minimal antibody uptake, and all nondraining LNs (e.g., axillary nodes, contralateral popliteal LN) were devoid of the s.c. delivered antibodies (Figure 1, C and D). The lymph-borne antibodies bound to their target antigens only in the first draining $\mathrm{LN}$ also after prolonged durations of in vivo distribution (up to 24 hours; Supplemental Figure 1C). Thus, lymph-borne antibodies are transferred through the subcapsular sinus floor to the parenchyma of the primary draining LN in a functionally intact form.

The uptake of lymph-borne antibodies into the parenchyma of the draining LN was a concentration-dependent process (Figure 2A and Supplemental Figure 1D). It was clearly detectable when 1-10 $\mu \mathrm{g}$ antibody was administered s.c. (and faintly with a $0.1-\mu \mathrm{g}$ dose). The transfer was extremely fast, since parenchymal staining by the lymph-borne antibodies was detectable even when the recipient mouse was sacrificed immediately after the injection (Figure 2B and Supplemental Figure 1E). When the same antibody pool was given i.v. (at 1- to 50- $\mu$ g doses), intravascular cells were labeled, but no staining was detectable in parenchymal cells outside the blood vessels (Supplemental Figure 2, A-C), indicating that BECs are unable to transfer antibodies through the vessel wall. The intranodal staining in the draining $\mathrm{LN}$ by the lymph-borne antibodies was not due to a possible leakage of free lymph-borne antibodies from the sinus during tissue processing, since untouched congenic lymphocytes added to the ex vivo-processing steps remained virtually unstained (Supplemental Figure 2 , D and E). Moreover, antibodies delivered in a $1-\mu \mathrm{l}$ volume $(2-\mu \mathrm{g}$ dose) were taken up very effectively to the parenchyma, implying that the injection pressure load was not affecting the transfer
Figure 2. Efficient isotype-dependent entry of s.c. administered antibodies into the draining LNs. (A and B) Flow cytometric analyses of the (A) dose dependency (fixed $t=5 \mathrm{~min}$ ) and (B) time dependency (1- $\mu \mathrm{g}$ fixed dose) of B220-PB and CD4-FITC (both of the IgG subclass) entry into the draining LN after s.c administration. (C) Confocal analyses of the distribution of an unconjugated IgM antibody (MECA79) in the draining $\mathrm{LN}$ after s.c. administration ( $5 \mu \mathrm{g}, t=5 \mathrm{~min}, n=4$ ). Ex vivo stainings (serial sections) with MECA-79 show the total pool of positive cells. Scale bars: $20 \mu \mathrm{m}$. HEV, high endothelial venule. (D) Flow cytometric analyses of lymphocytes in the draining LN after s.c. administration of CD8 antibodies of IgC2a and IgM isotypes (2- $\mu$ g dose, $t=30 \mathrm{~min}, n=3$ ). The cells were stained ex vivo for B220 and CD4. In the bar graphs, each dot represents $1 \mathrm{LN}$, and data are the mean $\pm \mathrm{SD}$. ${ }^{*} P<$ 0.05 and ${ }^{* *} P<0.001$, by Kruskal-Wallis $(\mathbf{A}$ and $\mathbf{B})$ and Mann-Whitney $U$ test (D).
(Supplemental Figure 2F). In fact, even 0.5- to 0.1- $\mu$ g doses of the antibody delivered s.c. in this small volume showed dose-dependent specific reactivity with the target cells (Supplemental Figure 2F). The antibody transfer took place in all 5 mouse strains studied (Figure 1, Supplemental Figure 2G, and data not shown). Thus, we found that s.c. administration of submicrogram quantities of antibodies led to their transfer into LN parenchyma within seconds.

Antibody transfer to the LN parenchyma is isotype dependent. Many of the biological functions of antibodies are dependent on the isotype of the molecule. We found that all tested lymphborne IgG antibodies (raised in mouse or any of the 6 other host species tested, both monoclonal and polyclonal antibodies, IgG1, IgG2a, IgG2b, and IgG2c subtypes, antibodies against any of the 18 tested surface antigens) entered the parenchyma of the draining LNs (Figure 1A, Supplemental Figure 3, A-I, and data not shown). IgG antibodies conjugated to any of the 12 tested fluorochromes (including FITC, Alexa Fluor, APC, PE, Pacific Blue, Brilliant Violet, eFluor, DyLight, and tandem conjugates), biotin, or HRP, as well as unconjugated antibodies were taken up in the LN (Figure 1A, Supplemental Figure 3, A-I, and data not shown), indicating that the transfer through the sinus floor was not induced by any particular fluorochrome. Notably, since the molecular mass of a PE-conjugated IgG molecule is close to $400 \mathrm{kDa}$, these experiments also revealed that relatively large molecules could be quickly transferred to the LN parenchyma (Supplemental Figure 3I). In sharp contrast, we observed that lymph-borne IgM antibodies were completely excluded from the parenchyma (Figure 2, C and D, and Supplemental Figure 3J), although they did gain access to the sinus (data not shown). Collectively, these data indicate that antibody binding to any sinusoidal cell type and antibody specificity in general are not decisive events in the uptake process and that the trans- 


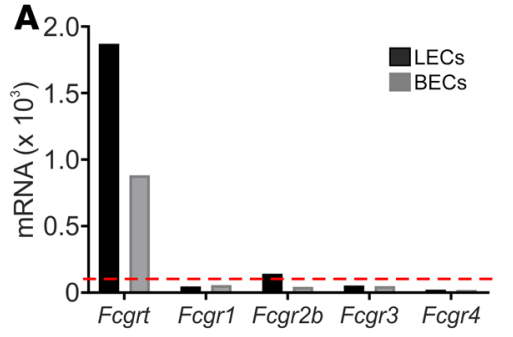

D

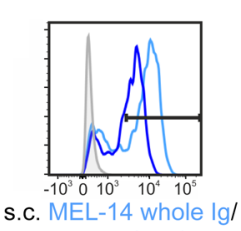
MEL-14(Fab)
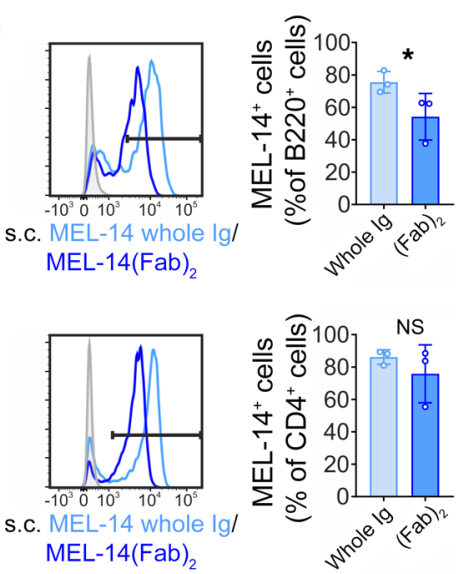

B
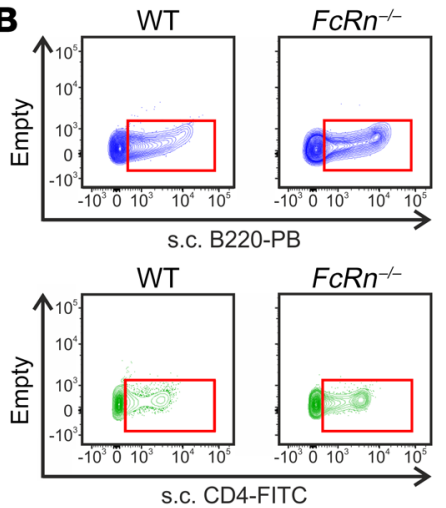

E
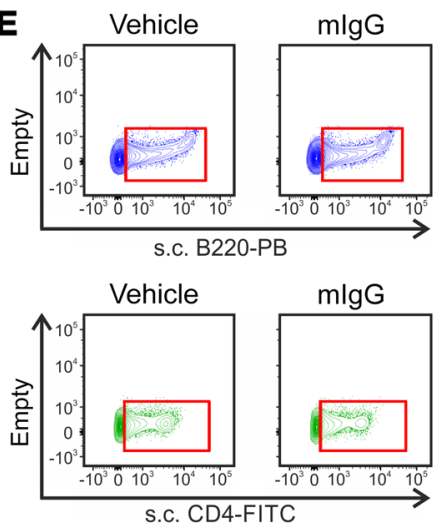
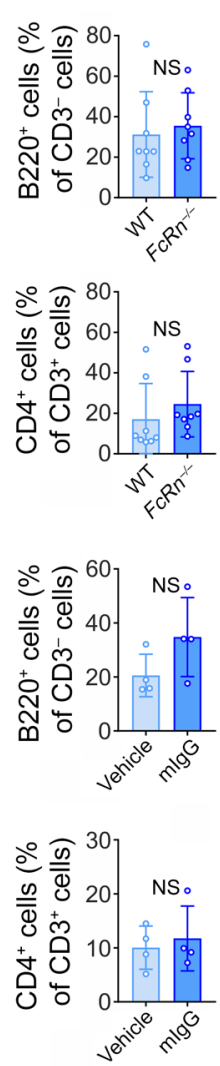

C
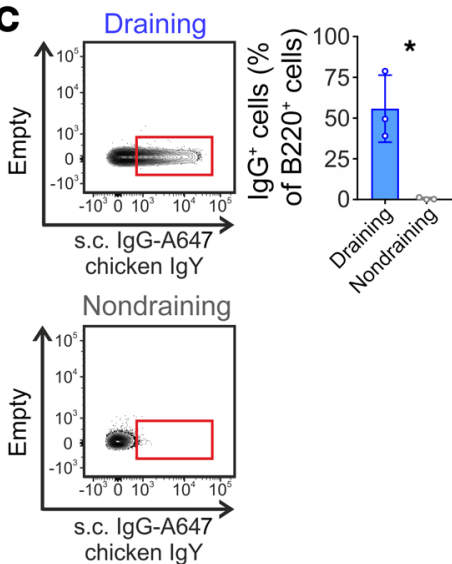

Figure 3. Fc tail- and Fc receptor-independent uptake of lymph-borne antibodies in draining LNs. (A) mRNA expression of Fc receptors in LECs and BECs in peripheral LNs (from Immunological Genome Project Data; https://www.immgen.org/). Values (mean) above 120 (red dashed line) indicate positivity with $95 \%$ confidence. (B) Flow cytometric analyses of lymphocytes in the draining LNs of WT and FcRn ${ }^{-1-}$ mice after s.c. administration of the indicated fluorochrome-conjugated B220 and CD4 antibodies (1- $\mu \mathrm{g}$ dose, $t=5 \mathrm{~min}, n=8)$. The cells were stained ex vivo for CD3. (C) Flow cytometric analyses of lymphocytes in the draining and nondraining LNs of WT mice after s.c. administration of chicken IgY (Alexa Fluor 647-conjugated IgY antibody against mouse IgG [IgG-A647]; $2 \mu \mathrm{g}, t=5 \mathrm{~min}, n=3$ ). The cells were stained ex vivo for B220. (D) Flow cytometric analyses of lymphocytes in the draining LNs of WT mice after s.c. administration of whole Ig and (Fab), fragments of the L-selectin antibody MEL-14 (2 $\mu \mathrm{g}, t=30 \mathrm{~min}, n=3$ ). The cells were stained ex vivo with Alexa Fluor 488-conjugated anti-rat IgG and for B220 and CD4. (E) Analyses of lymphocytes in the draining LNs of WT mice after s.c. administration of the indicated fluorochrome-conjugated B220 and CD4 antibodies ( $2 \mu \mathrm{g}, t=5 \mathrm{~min}, n=4)$ in the presence of a 100-fold excess of unlabeled polyclonal mouse IgG (mlgG) or vehicle control. The cells were stained ex vivo for CD3. In the bar graphs, each dot represents $1 \mathrm{LN}$, and data are the mean $\pm S D$. ${ }^{*} P<0.05$, by Mann-Whitney $U$ test.

fer through the sinus floor readily takes place for monomeric $\operatorname{Ig} G$ molecules and antibody conjugates but not for the pentameric IgM.

Antibody transfer through the sinus floor is a receptor-independent process. To identify the molecules carrying lymph-borne antibodies across the subcapsular sinus in LNs, we first focused on the neonatal Fc receptor ( $\mathrm{F} R \mathrm{Rn}$, also known as Fcgrt), which mediates the transfer of IgG, but not IgM, through the placenta and neonatal gut epithelium (15). Although Fcgrt mRNA was highly expressed in LECs in peripheral LNs, the transfer of s.c. administered antibodies was completely intact in $F c R n$-deficient $\left(F c R n^{--}\right)$mice (Figure 3, A and B, and Supplemental Figure 4A). FcRIIb (also known as Fcgr2b), expressed at low levels in LN LECs (Figure 3A) was not involved in the antibody transfer either, since a function-blocking antibody against this Fc receptor (16) did not interfere with the transfer process (Supplemental Figure 4B). Deglycosylation of the IgG Fc tail by PNGase F treatment from all N-linked oligosaccharides, which regulate FcR and complement binding (17), did not affect the parenchymal transfer of lymph-borne antibodies (Supplemental Figure 4, C and D, and see the complete, unedited blots in the supplemental material). Moreover, lymph-borne IgA molecules were taken up in the draining LNs (Supplemental Figure 4E). Notably, lymph-borne chicken IgY, which does not bind to any mammalian Fc or complement receptor (18), was also transferred effectively to the parenchyma (Figure 3C). These data indicate that the antibody transfer through the sinusoidal floor was independent of $\mathrm{Fc}$ and complement receptors.

To study the possible non-Fc receptor-dependent functions of the Fc tail in the antibody transfer, we compared the uptake of whole IgG and (Fab) ${ }_{2}$ fragments in the draining LNs. The (Fab) fragment entered the node parenchyma as efficiently as the full-length antibody (although the staining intensity was fainter because of the lower reactivity of the secondary antibody with the Fc tail-less antibody) (Figure 3D and Supplemental Figure 4F). Another (Fab) ${ }_{2}$ fragment and a Fab antibody were also transferred across the sinus when administered s.c. (Supplemental Figure $4, \mathrm{G}$ and $\mathrm{H}$ ). Finally, to study whether the transfer is a receptordependent process in the first place, we coadministered a 100 -fold excess of an unlabeled polyclonal mouse Ig or unlabeled mono- 
A
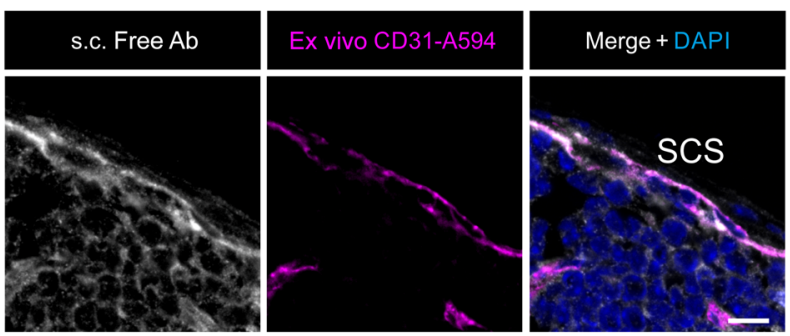

B
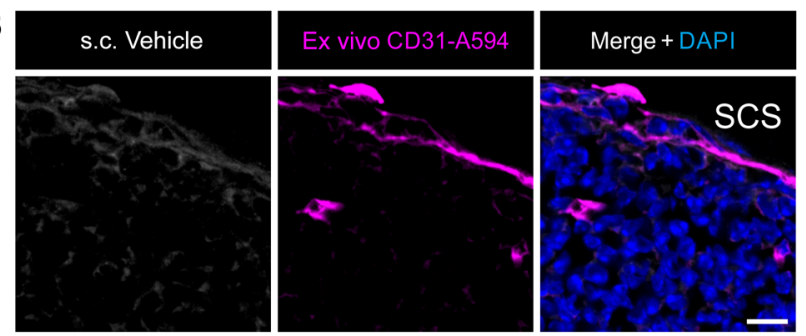

C
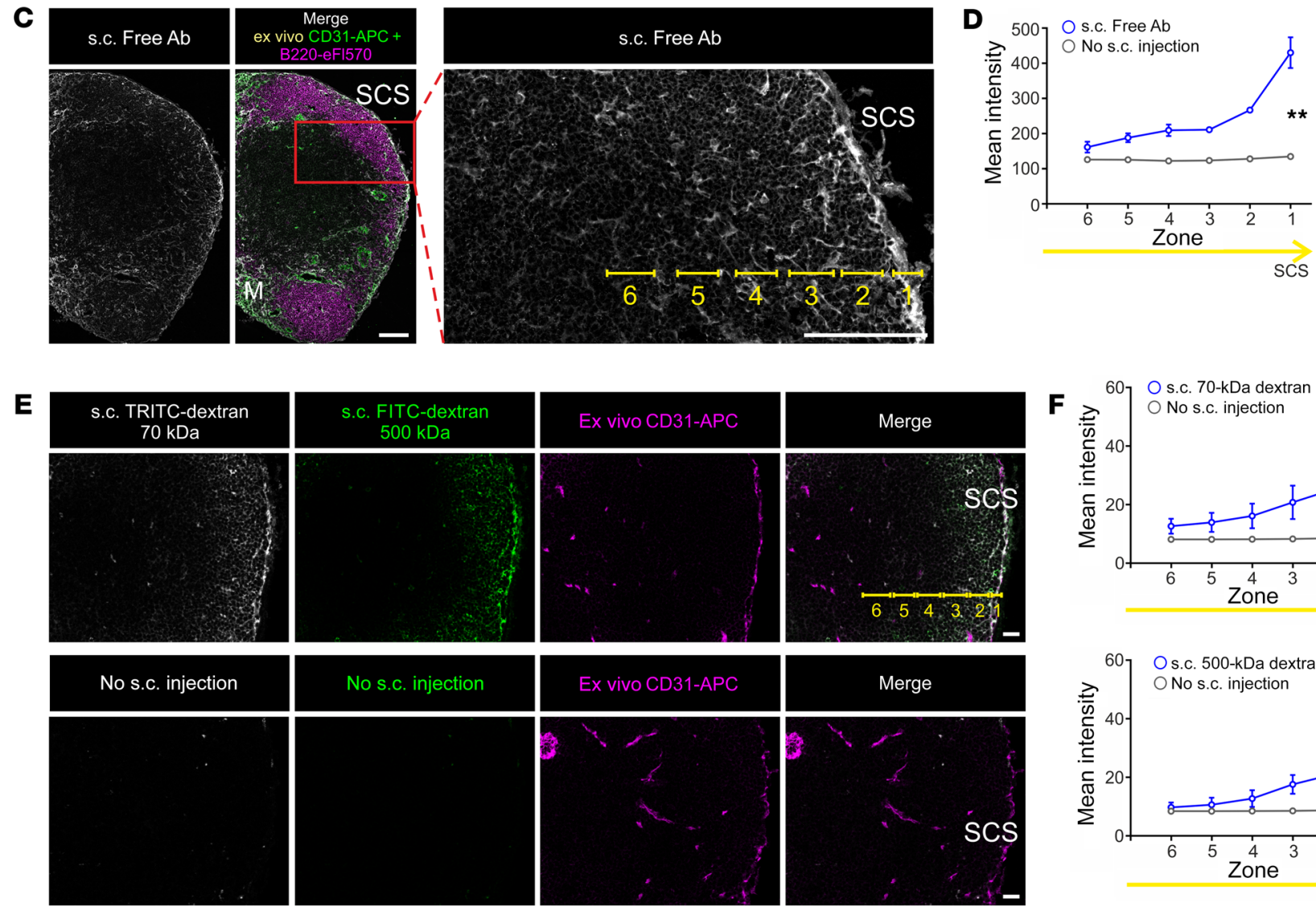

Figure 4. Fluid-phase transfer of Igs and dextrans to the parenchyma of draining LNs. (A and B) Confocal analyses of the subcapsular sinus of the draining LN after s.C. administration of (A) a nonbinding rat IgG2a antibody (free Ab, 5 - $\mu$ g dose, $t=2$ minutes, $n=3$ ) or (B) vehicle ( $t=2$ min, $n=4)$. The sections were stained ex vivo with an Alexa Fluor 488-conjugated anti-rat IgC (to detect the free Ab) and for CD31. Scale bars: $10 \mu \mathrm{m}$. (C and $\mathbf{D})$ Confocal analyses (C) and quantification ( $\mathbf{D}$, mean \pm SD of 3 measurements) of the free antibody signal ( 5 - $\mu$ g dose, $t=2$ min, $n=3$ ) from the indicated subsinusoidal zones (yellow indicates zone 1, representing the subcapsular sinus) in the draining LN after s.c. administrations. The sections were stained ex vivo for CD31 and B220. Scale bars: $100 \mu \mathrm{m}$. (E and F) Confocal analyses (E) and quantification (F, mean \pm SD of 4 measurements) of the dextran signal (50- $\mu$ g dose, $t=1$ $\min , n=2$ ) from the indicated subsinusoidal zones in the draining LN after s.c. administration of TRITC-labeled 70-kDa dextran and FITC-labeled 500-kDa dextran. No s.c. injection, a noninjected control LN. The sections were stained ex vivo for CD31. Scale bars: $20 \mu \mathrm{m}$. ${ }^{*} P<0.01$, by linear regression analysis.

clonal antibody with the labeled antibodies. Strikingly, the transfer of the indicator antibodies to the LN parenchyma remained completely intact in these competition experiments (Figure 3E and Supplemental Figure 4I). Thus, the noncompetitive, receptorindependent nature of the antibody uptake into the LN parenchy$\mathrm{ma}$ is indicative of a fluid-phase process.

Fluid-phase transfer of antibodies and other large biomolecules through the subcapsular sinus floor. To further address the molecular mechanisms of the antibody transfer to the LN parenchyma, we used a nonbinding unconjugated rat IgG2a isotype control antibody (hereafter referred to as free antibody), the transit of which is not affected by binding to any antigen in the LN. After s.c. administration of the free antibody, we fixed the draining LN by in situ treatment with paraformaldehyde and then visualized the distribution of the free lymph-borne antibody by adding a labeled secondary antibody ex vivo onto the tissue sections. We found that the free antibody localized to the cells of the sinus floor (Figure 4, A and B). In addition, the free antibody displayed a clear centrifugal concentration gradient in the node parenchyma, with the highest levels detected immediately below the sinus floor (Figure 4, C and D). Moreover, the free antibody apparently surrounded parenchymal lymphocytes in the cortical area of the draining LN 
A

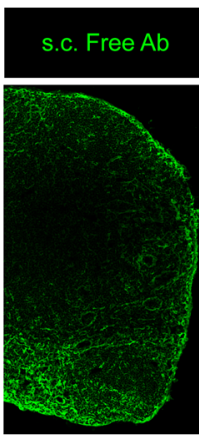

C
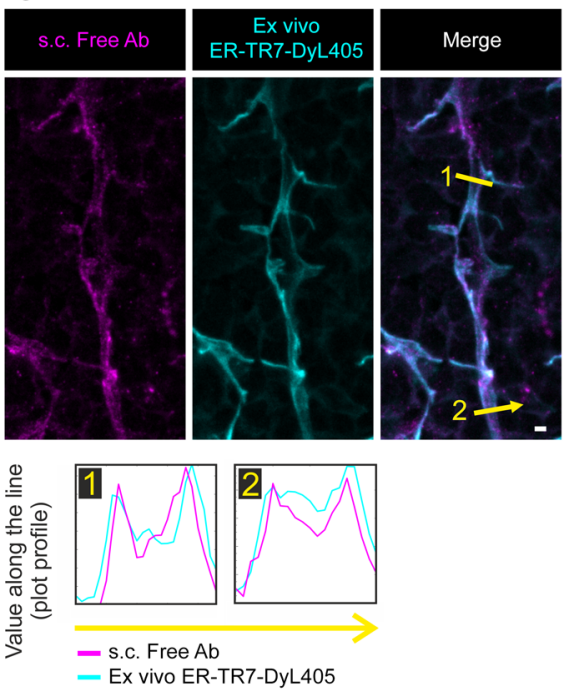

D
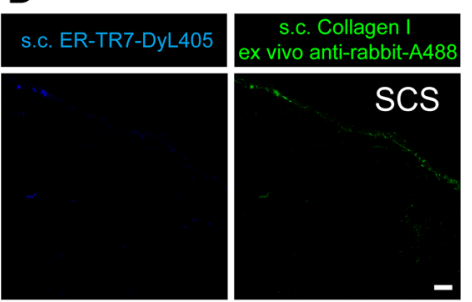
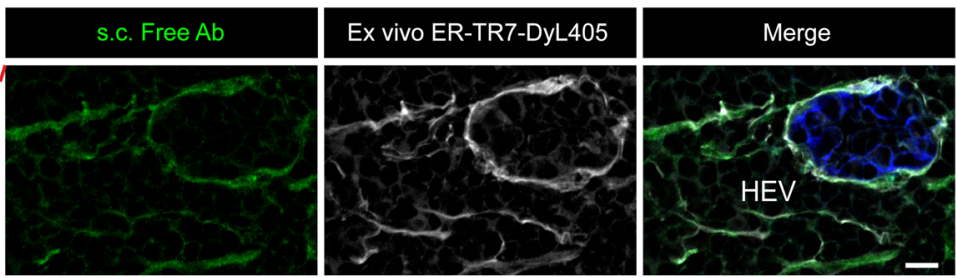

B

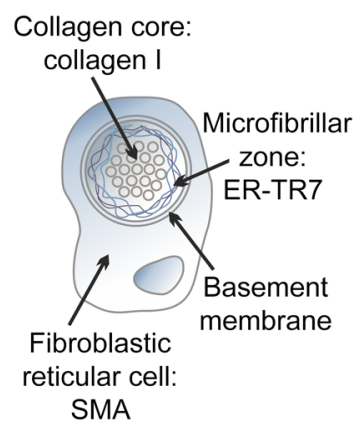

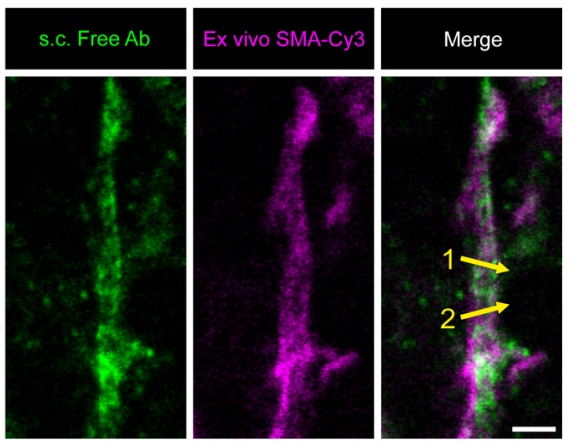
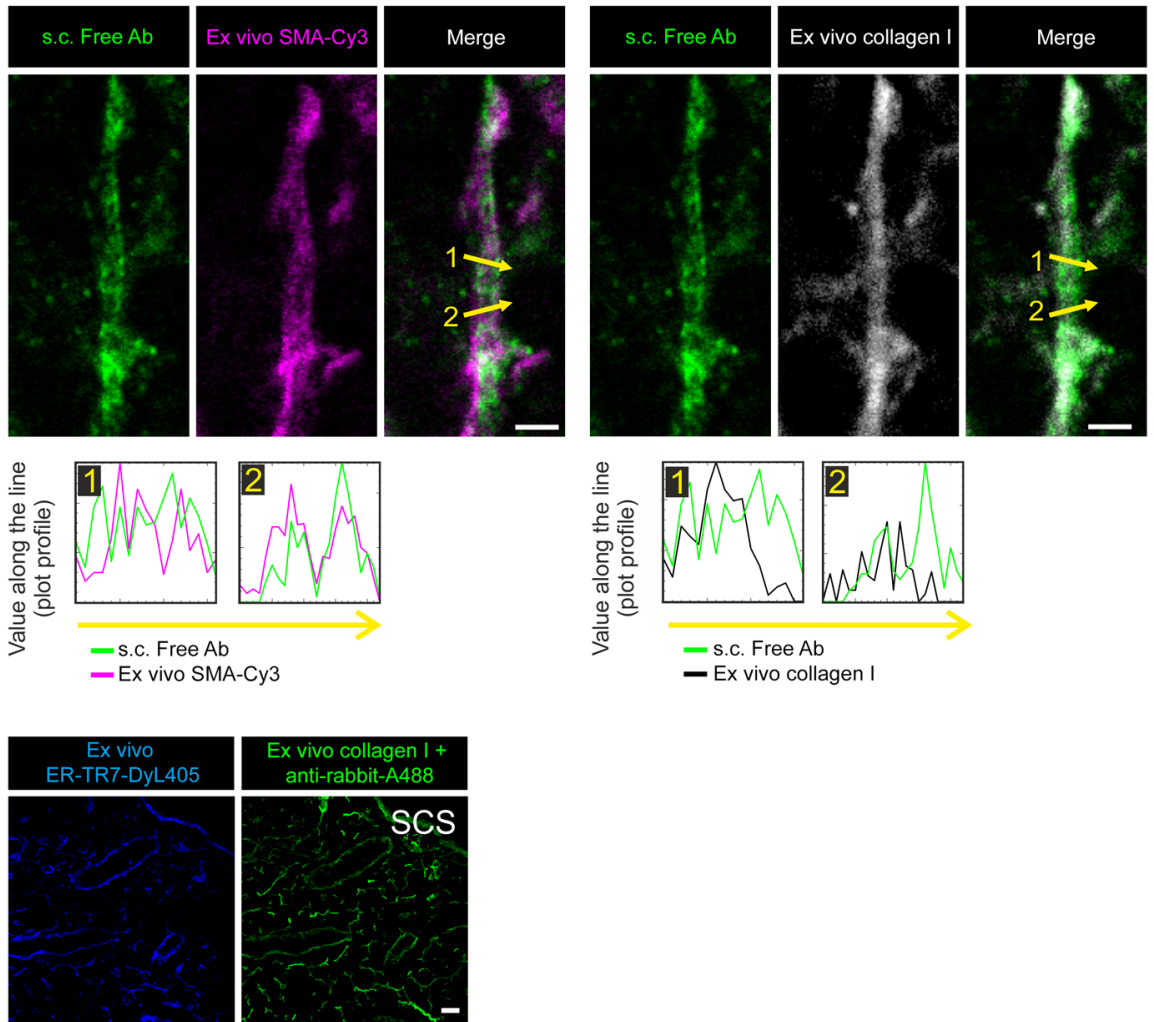

Figure 5. Transfer of lymph-borne antibodies through the sinus floor is independent of reticular conduits. (A) Confocal analyses of the conduits of paraformaldehyde-fixed draining LNs after s.c. administration of a nonbinding rat IgG2a antibody (free Ab; 5 - $\mu$ g dose, $t=1$ min, $n=3$ ). The sections were stained ex vivo with Alexa Fluor 488-conjugated anti-rabbit IgC (to detect the free Ab) and for CD31, B220, and ER-TR7. Scale bars: 100 $\mu \mathrm{m}$ and 20 $\mu \mathrm{m}$ (zoom). (B) Structure of the conduits and the marker antigens collagen I, ER-TR7, and SMA. (C) Confocal analyses of the conduits of paraformaldehyde-fixed draining LNs after s.c. administration of a nonbinding rat IgC2a antibody (free Ab; 5 - $\mu$ g dose, $t=1$ min, $n=3$ ). The sections were stained ex vivo with Alexa Fluor 488-conjugated anti-rat IgG (to detect the free Ab) and for ER-TR7, collagen I, and SMA and analyzed for the staining intensities across the conduits (yellow lines 1 and 2 ). Scale bars: $2 \mu \mathrm{m}$. ( $\mathbf{D}$ and $\mathbf{E}$ ) Confocal analyses of the conduits of nonfixed draining LNs (D) after s.C. administration of collagen I and ER-TR7 antibodies (both at a 2 - $\mu$ g dose, $t=5 \mathrm{~min}, n=3$ ) and (E) after ex vivo staining with the same antibodies ( $n=3$ ). Scale bars: $20 \mu \mathrm{m}$.

(Figure 4, A and C). These findings would be compatible with the presence of the transferred free antibody in the interstitial fluid and thus strongly argue for the fluid-phase nature of antibody transfer through the sinus floor.

Given that a fluid-phase transfer should not discriminate between antibodies and other soluble molecules of a similar size, we evaluated the movement of lymph-borne dextrans to the LN parenchyma. Fluorescently tagged 70-kDa dextran, which does not penetrate into the reticular conduits $(7,11)$, was also found intranodally after s.c. administration (Figure 4E). Moreover, upon s.c. injections, even a 500-kDa dextran was readily visible in the LN parenchyma surrounding lymphocytes (Figure 4E). In quantitative analyses, both 70-kDa and 500-kDa dextrans also showed centrifugal concentration gradients in the parenchyma of draining LNs (Figure 4F). Collectively, these data suggest that after transfer across the subcapsular sinus floor, the lymph-borne antibodies and other biomolecules spread intranodally by convection in the interstitial fluid.

The parenchymal entry of lymph-borne antibodies is an extraconduit and macrophage-independent process. The 2 known pathways mediating delivery of intact molecules to the parenchyma of 

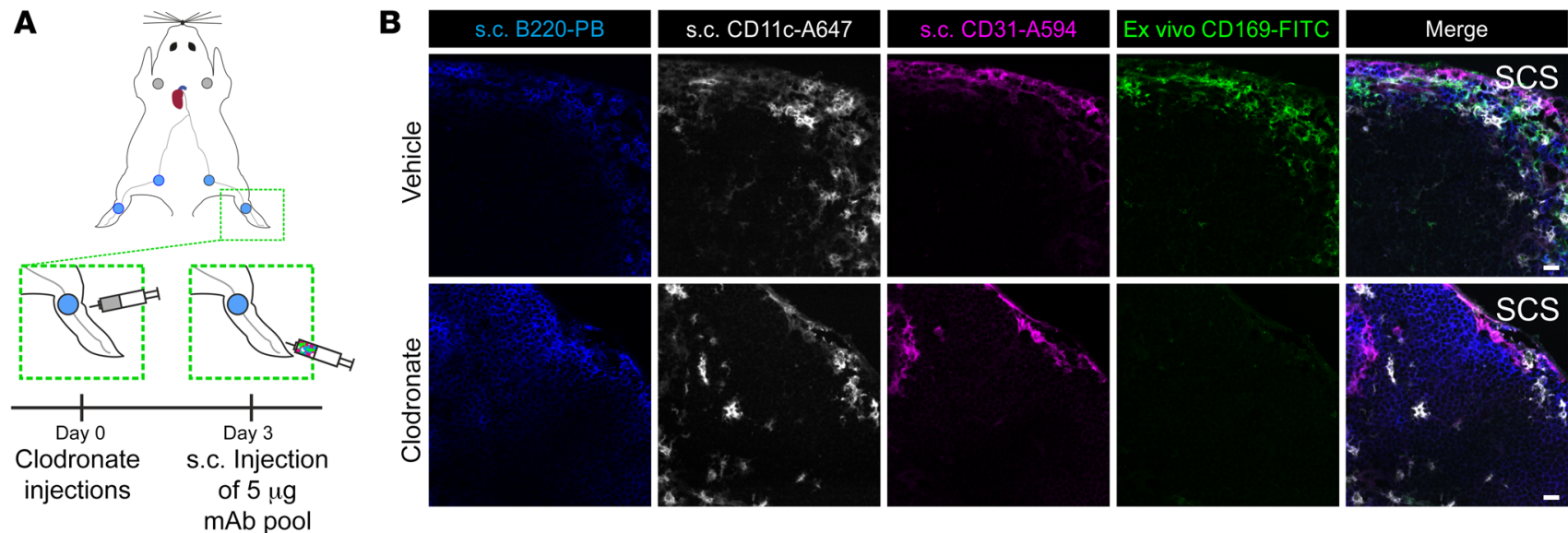

Figure 6. Subcapsular sinus macrophages are not involved in the transfer of antibodies through the sinus floor. (A) Experimental outline for selective depletion of subcapsular sinus macrophages. (B) Confocal analyses of clodronate- or vehicle-treated draining LNs after s.c. administration of fluorochrome-conjugated B220, CD11c, and CD31 antibodies (1- $\mu$ g dose, $t=1 \mathrm{~min}, n=3$ ). The sections were stained ex vivo for CD169, a marker for subcapsular sinus macrophages. Scale bars: $20 \mu \mathrm{m}$.

draining LNs are reticular conduits and subcapsular sinus macrophages. The reticular conduit system only allows the parenchymal entry of soluble lymph-borne molecules smaller than $70 \mathrm{kDa}$ in size (7-11). However, we observed that the lymph-borne free antibody was apparently concentrated along the reticular conduit network and blood vessels in the paraformaldehyde-fixed draining LN (Figure 5A). The conduits have an inner core composed of collagen I fibrils surrounded by concentric layers of a microfibrillar zone, basement membrane, and an outer wrapping of fibroblastic reticular cells (Refs. 9, 10, 12, and Figure 5B). Quantitative analyses revealed apparent colocalization of the free antibody with the microfibrillar zone marker ER-TR7 and the fibroblastic reticular cell marker smooth muscle actin (SMA) (Figure 5C). However, the free antibody apparently did not colocalize with the inner core marker collagen I (Figure 5C). To address the potential entry of antibodies into conduits using a functional assay, we injected the ER-TR7 and collagen I antibodies s.c. into mice. We reasoned that if antibodies entered the conduits, they should stain the internal conduit structures. On the other hand, if the antibodies entered the parenchyma outside the conduits, they should not be able to bind to the inner components of the conduits, which are tightly ensheathed by the fibroblastic reticular cells and basement membranes $(19,20)$. When we analyzed nonfixed LNs after the s.c. injections of these 2 antibodies, we found no reactivity with the conduits (Figure 5D). Importantly, in control experiments, the same antibodies reacted strongly with the conduits when directly applied to the sections ex vivo (Figure 5E). These data suggest that the transcytosed free antibody was apposed to but not within the conduits. The free antibody was also concentrated around the ER-TR7 ${ }^{+}$basal aspects of high endothelial venules (Figure 5A). Collectively, these findings indicate that after lymph-borne antibodies have been transferred through the sinus floor, they move freely around the cellular constituents and matrix barriers in the parenchyma of the draining LNs.

Ready-made immunocomplexes have been reported to enter the LN parenchyma by propelling on the surface of subcapsular sinus macrophages $(21,22)$. To analyze the potential role of sub- capsular sinus macrophages in the transfer of antibodies through the sinus floor, we locally depleted this cell type by injecting clodronate into the calf of recipient mice (Figure 6A). This treatment protocol leaves the footpad intact for subsequent injections (23). CD169 staining verified an effective depletion of subcapsular sinus macrophages in the draining popliteal LNs of clodronate-treated legs, whereas these cells remained intact in the vehicle-treated legs (Figure 6B). Notably, the absence of subcapsular sinus macrophages did not affect the parenchymal transfer of lymph-borne B220, CD11c, or CD31 antibodies in any way (Figure 6B). Thus, the extra-conduit and macrophage-independent nature of antibody transfer through the sinus floor is strongly indicative of a new mode of lymph filtration.

Transcytosis of lymph-borne antibodies through subcapsular sinus LECs. The floor of the subcapsular sinus is mainly composed of LECs interspersed with subcapsular sinus macrophages (refs. 1, 11, 24, and Supplemental Figure 5, A and B). Since macrophages were not involved in the antibody transfer, we next focused on the potential role of LECs in the uptake of antibodies. Certain macromolecules can be shuttled from one surface of the cell to the other by transcellular vesicular transport, i.e., transcytosis (15, 25-30). We found that the extremely attenuated (often $<200 \mathrm{~nm}$ in thickness) cytoplasmic projections of sinusoidal LECs are very rich in heterogeneous cytoplasmic vesicles resembling flask-shaped caveolae, round intracytoplasmic vesicles, and elongated tubulovacuolar structures (Figure 7A). Immunoelectron microscopy revealed that the lymph-borne free antibody localized to heterogeneous vesicular and tubular structures in the LECs of the sinus floor (Figure 7, B-D, and Supplemental Figure 5, C-E). The antibody did not induce the formation of any macropinocytic vesicles (Figure 7, B-D, and Supplemental Figure 5, C-E), and the inter-EC junctions remained intact and devoid of the antibody signal (Figure 7E).

To investigate the endosomal nature of the free antibodycontaining vesicles, we performed Airyscan high-resolution confocal imaging of EEA1 ${ }^{+}$vesicles (a marker of early endosomes; ref. 31) in the subcapsular sinus floor. When focusing on $\mathrm{CD} 31^{+}$ floor LECs, we found multiple clearly identifiable EEA1 ${ }^{+}$early 


\section{A}

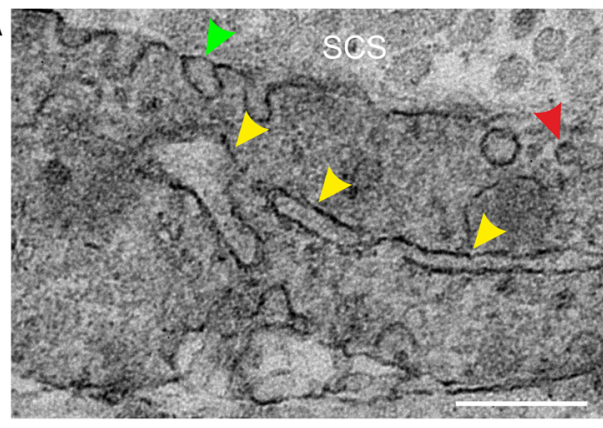

B

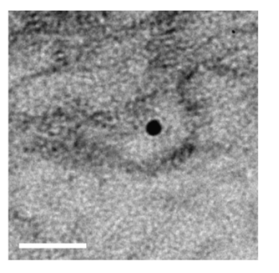

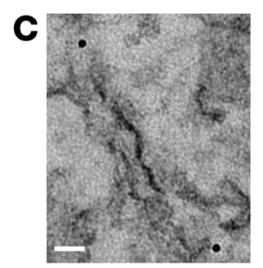
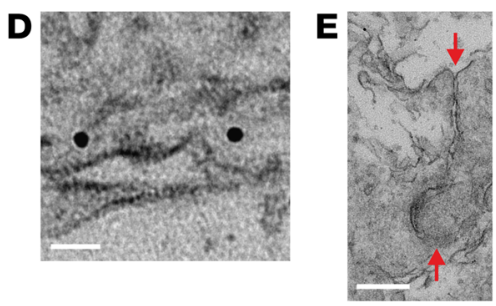

$\mathbf{F}$

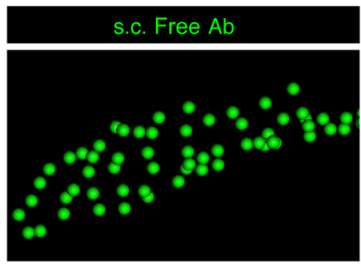

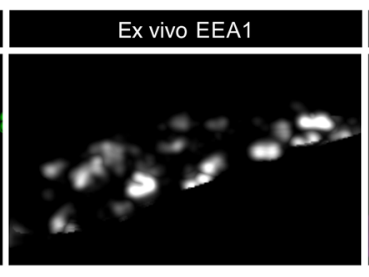

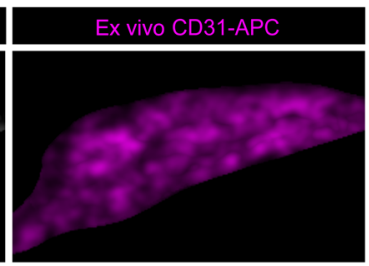

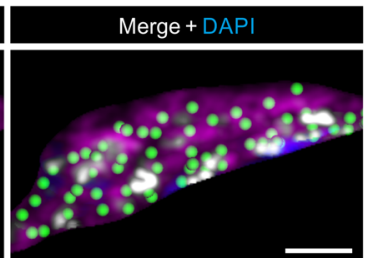

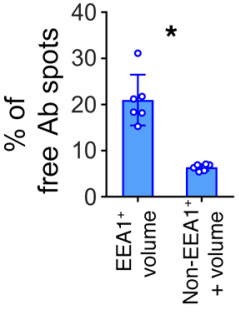

Figure 7. Vesicular transcytosis of lymph-borne antibodies through sinusoidal LECs in the draining LNs. (A) Transmission electron microscopic analyses of sinusoidal LECs $(n=3)$. Green arrowhead indicates a caveola; red arrowhead indicates round intracytoplasmic vesicles; yellow arrowheads indicate elongated tubulovesicular structures. Scale bar: $200 \mathrm{~nm}$. (B-E) Immunoelectron microscopic analyses of the localization of lymph-borne free antibody $(0.5-\mu \mathrm{g}$ dose, $t=30 \mathrm{~min}, n=2)$ in the cytoplasmic vesicles of sinusoidal ECs $(\mathbf{B}-\mathbf{D})$ and at inter-EC junctions (red arrows in $\mathbf{E})$ in the draining LN. The sections were stained ex vivo with anti-rat IgG antibody conjugated to $10-\mathrm{nm}$ gold particles. Scale bars: $40 \mathrm{~nm}$ (B-D) and $200 \mathrm{~nm}$ (E). (F) Confocal high-resolution analysis of the lymph-borne free antibody ( 2 - $\mu$ g dose, $t=5 \mathrm{~min}, n=6$ images from 3 individual popliteal LNs) in the early endosomes of floor LECs. The sections were stained ex vivo for CD31 and EEA1, and the distribution of free antibody vesicles in contact with EEA1 ${ }^{+}$vesicles and localized to similar non-EEA $1^{+}$volumes (normalized to the cell volume) was determined. Scale bar: $1 \mu \mathrm{m}$. In the bar graphs, data are the mean \pm SD. ${ }^{*} P<0.05$, by Wilcoxon matched-pairs, signed-rank test.

endosomes (Supplemental Figure 5F) and observed that the free antibody was localized within or in contact with a number of EEA1 ${ }^{+}$endosomes (Figure 7F and Supplemental Video 1) and was enriched in EEA1 ${ }^{+}$volumes when compared with EEA1 ${ }^{-}$volumes within the LECs. Collectively, the immunoelectron microscopy and confocal imaging data indicate that antibody transfer from lymph to the parenchyma of the draining LN occurs via transcytosis through sinusoidal LECs.

To mechanistically study the endocytosis and transcytosis of antibodies in the floor LECs, we functionally interfered with these pathways using chemical inhibitors and, when available, gene-deficient mice. Transcytosis in BECs is largely mediated by caveolae $(15,26-29)$. We therefore studied antibody transcytosis in caveolin-1-deficient mice (Cav1-/- mice), which lack all caveolae and hence caveolin-dependent endocytosis and transcytosis (32). However, we found that the transcytosis of lymph-borne antibodies was completely intact in the absence of caveolae (Figure 8A). To dissect the role of other endocytic pathways in the antibody transfer, we topically applied selective endocytosis inhibitors directly to surgically exposed draining LNs, leaving the footpad lymphatics unperturbed for s.c. antibody injections (Figure 8B). One leg of the mouse was always used for the inhibitor treatment and the other leg for the control treatment. We first focused on the major clathrin-mediated endocytosis pathway. Pitstop 2, a cell-permeable small-molecule inhibitor blocking ligand binding to the clathrin terminal domain (33), did not significantly inhibit transcytosis of CD4 or B220 antibodies when compared with a structurally similar Pitstop 2 negative control compound (Figure 8C and Supplemental Figure 6, A and B). We found that antibody transcytosis was also similar in the presence of vehicle and monodansylcadaverine, a classical inhibitor of clathrin-mediated endocytosis (ref. 34, Figure 8D, and Supplemental Figure 6, C and D). Among nonclathrin-mediated endocytic processes, macropinocytosis mediates the uptake of nutrients and extracellular fluid into cells (35). However, neither EIPA, an amiloride derivative inhibiting $\mathrm{Na}^{+} / \mathrm{H}^{+}$exchange needed for macropinosome maturation (36), nor imipramine, a newly identified selective inhibitor of macropinocytosis (37), interfered with antibody transfer (Figure 8, $\mathrm{E}$ and F, and Supplemental Figure 6, E-H).

Since many endocytosis routes are dynamin dependent, we tested the effect of dynamin inhibitors on the transfer of antibodies. Microscopy revealed that in Dyngo-4a-treated (38) LNs, lymph-borne B220 and CD31 antibodies did not penetrate as deeply into the parenchyma below the sinus and did not stain the cells as brightly as in the vehicle-treated controls (Figure 9A). Flow cytometric quantifications showed that the Dyngo-4a treatment inhibited approximately $70 \%$ of the antibody transfer and verified the lower staining intensity of the remaining cells (Figure 9B and Supplemental Figure 6I). Importantly, when using the local Dyngo4a application, delivery of the lymph-borne antibodies up to the subcapsular sinus remained intact, as revealed by sinusoidal EC staining by the CD31 antibody (Figure 9A). The use of another structurally distinct dynamin inhibitor, Dynole 34-2 (39), and its specific negative control compound, Dynole 31-2, confirmed the 


\section{A}
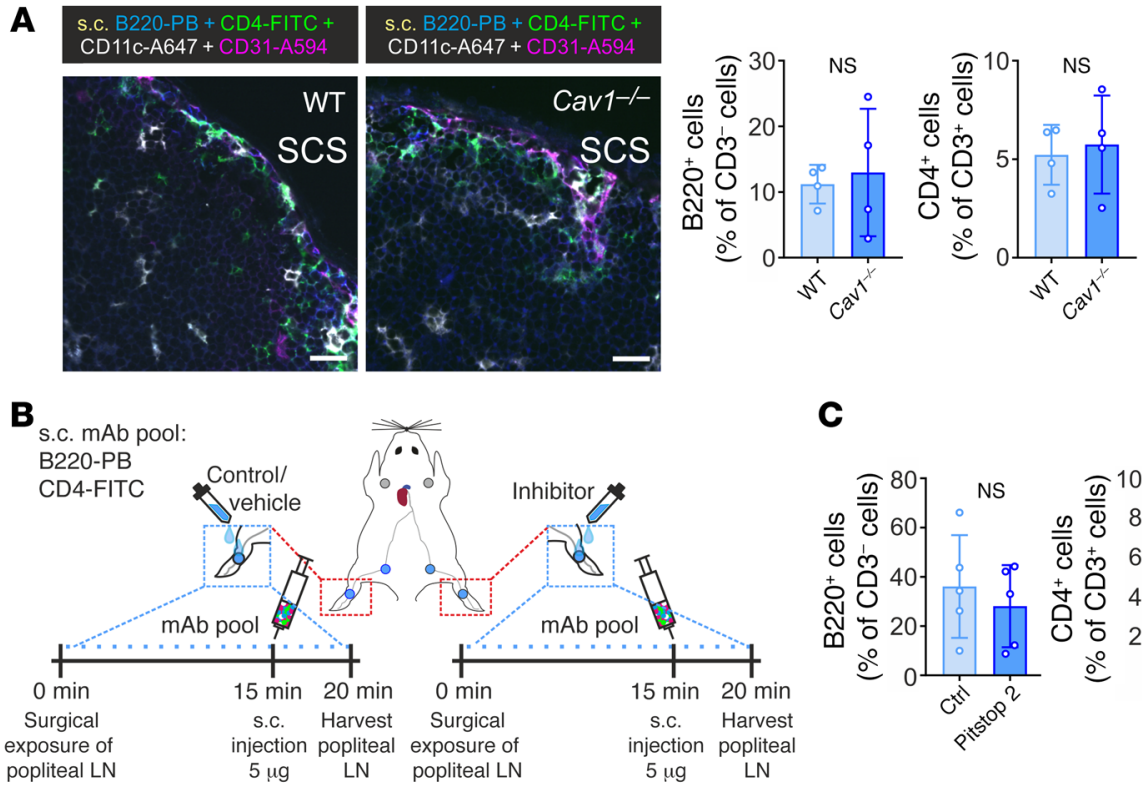

C

D

E

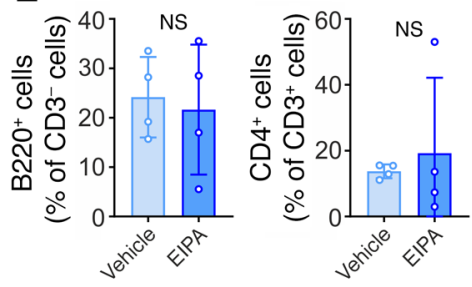

F

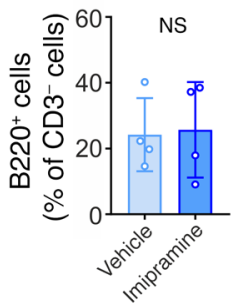

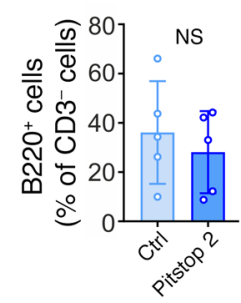

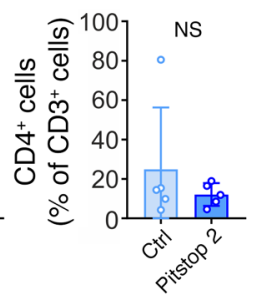

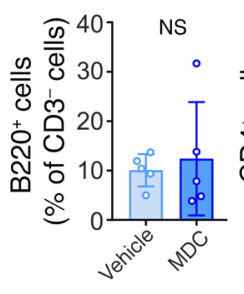

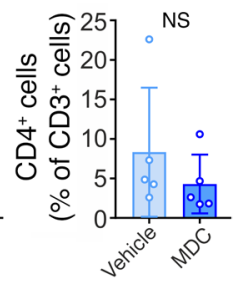

Figure 8. Analyses of the role of endocytosis routes and paracellular permeability in antibody transfer to the draining LNs. (A) Confocal and flow cytometric analyses of draining LNs of WT and Cav1 ${ }^{-1-}$ mice after s.c. administration of the indicated fluorochrome-conjugated antibodies ( $1 \mu$ g, $t=5$ min, $n=4)$. The cells were stained ex vivo for CD3 for flow cytometric analysis. Scale bars: $20 \mu m$. (B) Experimental outline for topical application of endocytosis inhibitors (and controls) to surgically exposed LNs. (C-G) Analyses of transcytosis of the indicated s.c. administered fluorochrome-conjugated antibodies ( $5 \mu$ g each, $t=5 \mathrm{~min}$ ) after topical pretreatment of the draining LNs with (C-F) endocytosis inhibitors or (G) a LEC junctional stabilizer. (C) Pitstop 2 (a clathrin inhibitor) and a Pitstop 2 negative control; (D) monodansylcadaverine (MDC) (a clathrin inhibitor) and vehicle; (E) EIPA (a macropinocytosis inhibitor) and vehicle; (F) imipramine (a macropinocytosis inhibitor) and vehicle; and (G) adrenomedullin and vehicle. The cells were stained ex vivo for CD3. In the bar graphs, each dot represents $1 \mathrm{LN}$, and data are the mean \pm SD. Statistical significance was determined by Mann-Whitney $U$ test.

dynamin dependence of the antibody transfer across the LN sinus (Figure 9C and Supplemental Figure 6J). Finally, we found that paracellular permeability was not likely to regulate the antibody transfer, since adrenomedullin, which stabilizes lymphatic endothelial junctions (40), had no detectable effect on the antibody transfer (Figure 8G and Supplemental Figure 6, K and L). Our imaging and functional data, thus, collectively indicate that the fluid-phase pinocytotic transcytosis of antibodies across the sinus is largely mediated by dynamin-dependent endocytosis and transfer in endomembranes and tubulovesicular structures of LECs.

Rapid ingestion of transcytosed large protein antigens by resident antigen-presenting cells in the LNs. Given their inability to enter the reticular conduits, large protein antigens from the periphery are thought to gain entrance into the parenchyma of the draining LNs only within migratory DCs (1, 2, 7-11). The uptake of antigens and DC activation and migration to the draining LN takes approximately 10 hours. We reasoned that the fluid-phase transcytosis pathway through the LECs of the sinus floor should deliver in seconds any large protein antigen to the draining $\mathrm{LN}$, where it can be processed by the local resident antigen-presenting cells. To experimentally test this, we s.c. administered a fluorescently tagged exogenous 180-kDa protein antigen (Alexa Fluor 647-labeled human AOC3 protein) and followed its uptake into the resident LN DCs and macrophages. We found that more than $15 \%$ of tissue-resident DCs $\left(\mathrm{CD} 45^{+} \mathrm{CD}^{-}{ }^{-} \mathrm{CD} 19^{-} \mathrm{CD} 11 \mathrm{c}^{\text {hi }} \mathrm{MHC} \mathrm{II}^{+}\right.$cells) (41) had ingested the antigen after 2 hours (Figure 10, A-C). As expected, the large protein antigen was also found in subcapsular sinus macrophages $\left(\mathrm{CD} 45^{+} \mathrm{CD}^{-} \mathrm{CD}^{-} 9^{-} \mathrm{CD} 11 \mathrm{c}^{-} \mathrm{CD} 11 \mathrm{~b}^{+} \mathrm{F} 4 / 80^{-} \mathrm{CD} 169^{+}\right.$ cells) and medullary sinus macrophages $\left(\mathrm{CD} 45^{+} \mathrm{CD} 3^{-} \mathrm{CD} 19^{-}\right.$

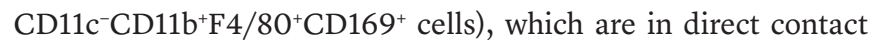
with the lymph in the sinus (Figure 10, A and D). Importantly, many LN parenchyma-resident macrophages $\left(\mathrm{CD}^{2} 5^{+} \mathrm{CD}^{-} \mathrm{CD} 19^{-}\right.$ $\mathrm{CD} 11 \mathrm{c}^{-} \mathrm{CD} 11 \mathrm{~b}^{+} \mathrm{F} 4 / 80^{+} \mathrm{CD} 169^{-}$cells) (6), which are not in contact with the sinus, had also taken up the s.c. delivered protein antigen within 2 hours (Figure 10, A and D). Collectively, these data show that large lymph-borne protein antigens from the periphery are transcytosed to the LN parenchyma and rapidly presented to the immune system well before the arrival of migratory DCs. 

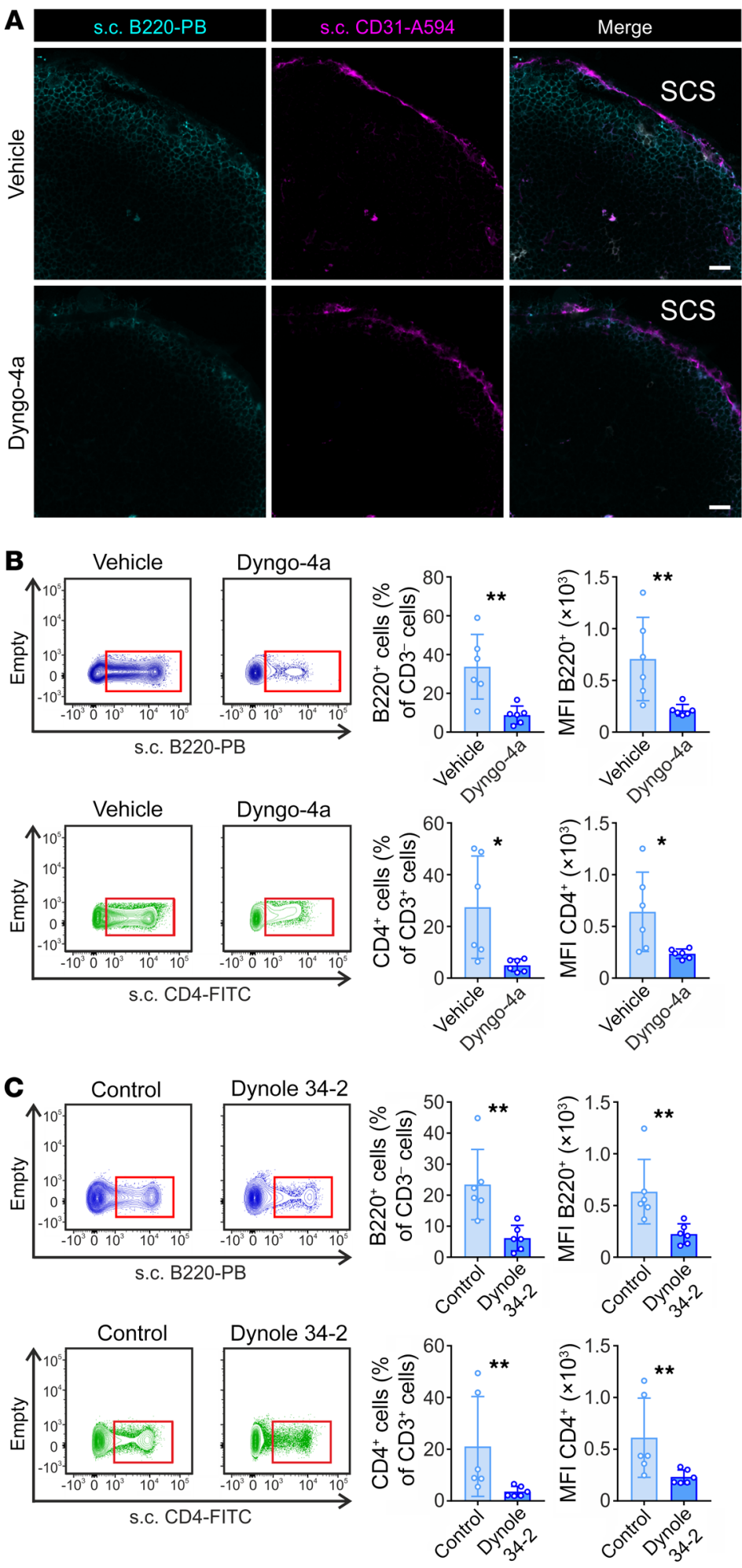

Improved bioimaging by application of parenchymal transfer of lymph-borne antibodies. After showing physiological relevance of the transfer of large lymph-borne proteins through the subcapsular sinus floor, we wanted to test whether this route could be harnessed for other purposes as well. Whole-mount imaging of optically cleared organs has been instrumental in dissecting the functional anatomy of tissues. However, the current techniques
Figure 9. Dynamin-dependent transcytosis of lymph-borne antibodies through floor LECs. Analyses of the transcytosis of the indicated s.c. administered fluorochrome-conjugated antibodies ( $5 \mu \mathrm{g}$ each, $t=5 \mathrm{~min}$ ) after topical pretreatment of the draining LN (as in Figure 8B) with (A and B) Dyngo-4a and vehicle and (C) Dynole 34-2 and the negative control Dynole 31-2. Scale bars: 20 $\mu \mathrm{m}(\mathbf{A})$. In $\mathbf{B}$ and $\mathbf{C}$, the cells were stained ex vivo for CD3. In the bar graphs, each dot represents $1 \mathrm{LN}$, and data are the mean $\pm \mathrm{SD} .{ }^{*} P<$ 0.05 and ${ }^{*} P<0.01$, by Mann-Whitney $U$ test.

suffer from loss of antigenic epitopes, poor performance of directly conjugated antibodies, and long staining times (typically 4 days) required for the penetration of antibodies into permeabilized tissues in vitro (42-46). We found that 30 minutes of in vivo staining with a pool of different fluorochrome-labeled antibodies followed by a short clearing allowed efficient 3D visualization of parenchymal cells in the draining LN when the staining antibodies were delivered in vivo by s.c. administration (Figure 11A and Supplemental Video 2). These analyses also confirmed that convection of antibodies into the B cell follicles was slower than that into the interfollicular stroma. Moreover, antibodies, which are exquisitely sensitive to target epitope destruction by any type of tissue processing, worked unperturbed in immunohistochemistry when allowed to be transferred in vivo into an intact draining $\mathrm{LN}$ through the sinus floor (Supplemental Figure 7, A and B). Thus, application of parenchymal transfer of lymph-borne antibodies for whole-mount imaging allows for single-step staining with pools of fluorochrome-conjugated antibodies combined with a more than 100-fold shortening of the staining time compared with current protocols.

Local control of immune responses by the transfer oflymphborne antibodies in vivo. Finally, we evaluated the potential of the parenchymal transfer of lymph-borne antibodies in controlling local immune activation. $T$ cell activation in vitro is routinely done by using a combination of plate-bound agonistic antibodies against the $\mathrm{T}$ cell receptor and $\mathrm{CD} 3$. We injected $2 \mu \mathrm{g}$ of these antibodies s.c. and found that they remained local in the draining LN throughout the duration of the experiment (Supplemental Figure 7, C and D). When the antibody-triggered induction of early $\mathrm{T}$ cell activation was analyzed after 18 hours, we found very efficient upregulation of $\mathrm{CD} 69$ and $\mathrm{CD} 25$ on the $\mathrm{CD} 4^{+}$and $\mathrm{CD} 8^{+} \mathrm{T}$ lymphocytes in the draining LNs, but not in the nondraining LNs (Figure 11B). Control antibodies did not trigger any lymphocyte activation (Supplemental Figure 7E).

Lymphocyte proliferation was also locally inducible when we used lymph-borne antibodies against immune checkpoint molecules. We transferred naive CFSE-labeled OVA-specific transgenic T lymphocytes (OTII cells) into WT recipient mice and allowed the cells to home to LNs. The next day, OVA was injected, and control and checkpoint-modulating antibodies were injected together with OVA and on the following 2 days. When analyzed on day 4 , we found that OVA and the control antibody induced modest proliferation (measured by CFSE dilution) of the transferred OTII cells in the draining LNs (Figure 11, 
A
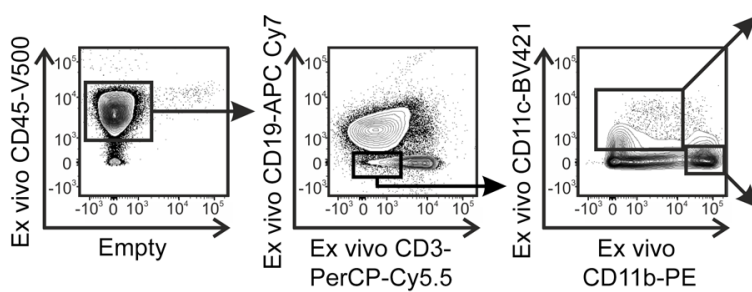

D
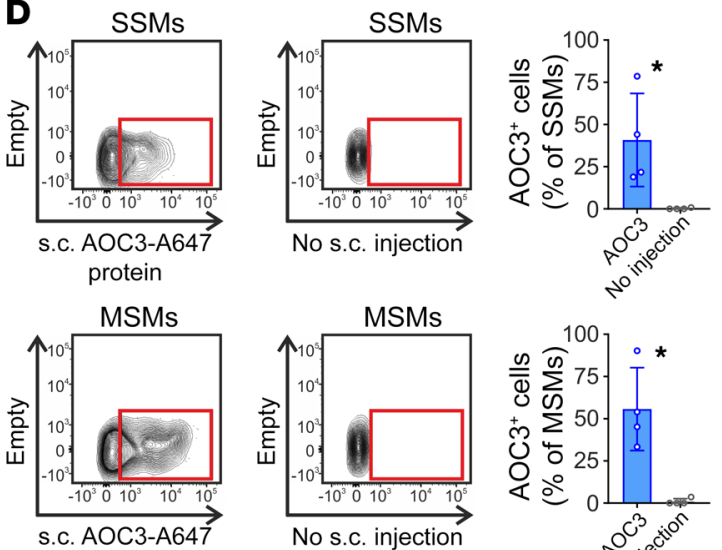

protein

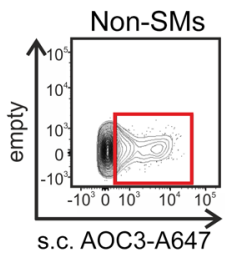

protein
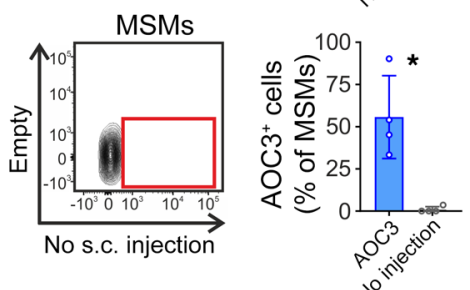

30
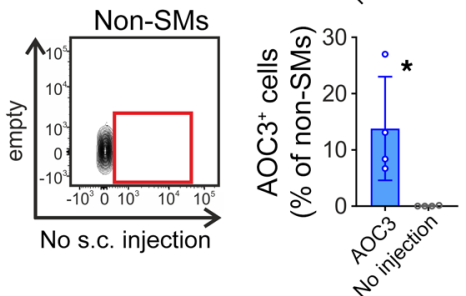

B
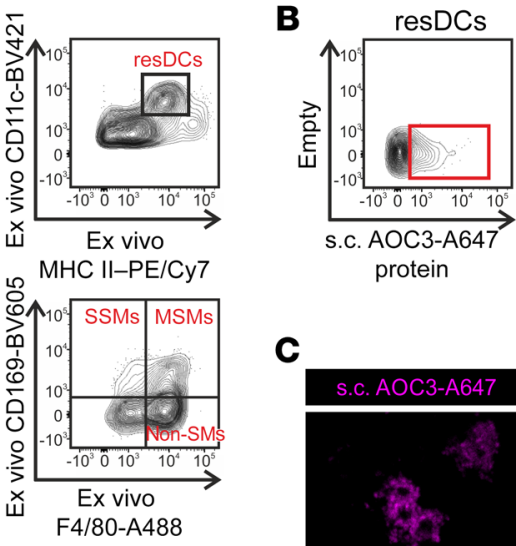

C
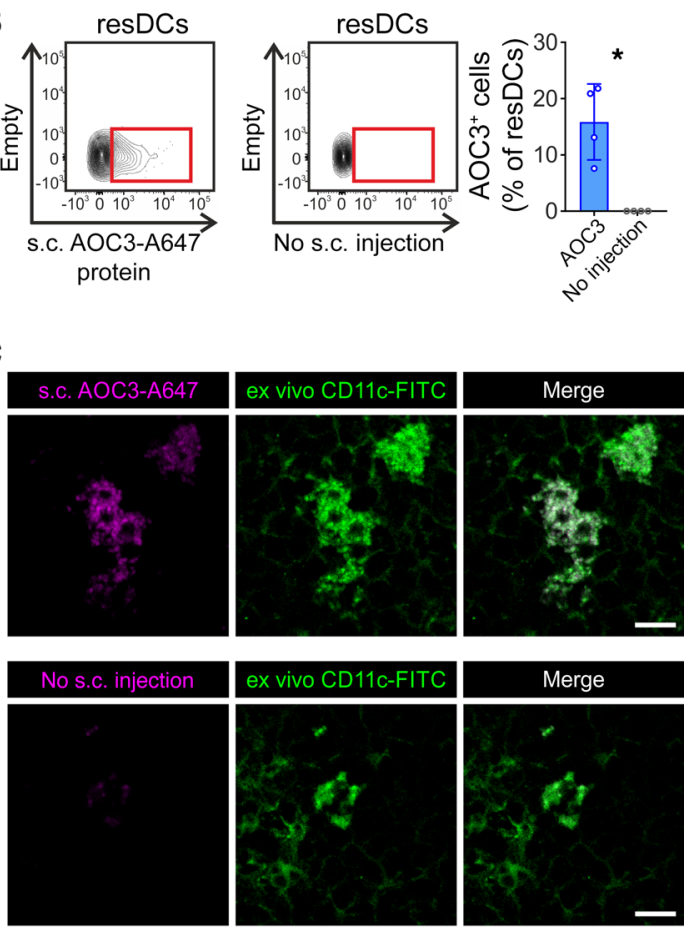

Figure 10. Fast delivery of large lymph-borne antigens to resident antigen-presenting cells in the draining LN. (A-D) Uptake of Alexa Fluor 647-labeled AOC3 antigen (180 kDa) after s.c. administration ( 2 - $\mu \mathrm{g}$ dose, $t=2 \mathrm{~h}$ ) in resident DCs (resDCs) and macrophages in the draining LN. Uninjected LNs served as negative controls. (A) Gating strategy for identification of resident DCs, medullary sinus macrophages (MSMs), and nonsinusoidal resident macrophages (non-SMs). (B and C) Flow cytometric analyses (B) $(n=4)$, confocal analyses (C) $(n=2)$, and quantification of AOC3 uptake in resident DCs. Scale bars: $10 \mu \mathrm{m}$. (D) Flow cytometric analyses and quantification of AOC3 uptake in resident macrophage populations $(n=4)$. In the bar graphs, each dot represents $1 \mathrm{LN}$, and data are the mean $\pm \mathrm{SD} .{ }^{*} P<0.05$, by Mann-Whitney $U$ test.
C and D). Coinjection of the agonistic costimulatory anti-CD28 antibody D665 (47) with OVA triggered vigorous cell division in the draining LNs, but not in nondraining LNs (Figure 11, C and D). When the agonistic (D665) (48) CD28 antibody was s.c. administered to the left footpad and the antagonistic (37.51) (49) CD28 antibody was injected into the right footpad of the same animal, we found robust lymphocyte proliferation in the left draining $\mathrm{LN}$ when compared with the right draining LN (Figure 11, E-G). Thus, local delivery of s.c. administered antibodies through the sinus floor allows functional manipulation of lymphocyte activation in a spatially controlled manner in vivo.

\section{Discussion}

Here, we have identified a transcytosis mechanism for lymph filtering in draining LNs. We show that large, intact lymph-borne biomolecules, including s.c. administered antibodies, are transferred across the subcapsular sinus floor to the parenchyma of the draining LN in seconds. We believe this profoundly alters the current dogma that large $(>70 \mathrm{kDa})$ foreign antigens from the periphery only gain entrance into the LN parenchyma approximately 10 hours after proteolysis within migratory DCs. We found that parenchymal transfer of large biomolecules takes place via a previously unknown fluid-phase, dynamin-dependent transcyto- sis pathway through sinusoidal LECs. We also show that in vivo antibody uptake through the sinusoidal floor can be utilized to improve bioimaging and for local modulation of immune responses in draining LNs.

Two main mechanisms mediating protein transfer across the subcapsular sinus in the draining LN have been described. Soluble lymph-borne molecules with a hydrodynamic radius of less than 5 to $6 \mathrm{~nm}(<70-\mathrm{kDa}$ globular proteins) enter the LN parenchyma via the reticular conduit network, which excludes all larger molecules (7-11). On the other hand, ready-made immunocomplexes are selectively delivered to follicular B cells in a complementreceptor-dependent manner by subcapsular sinus macrophages (21, 22). The transcytosis process we have identified here is completely different from these 2 previously described transport mechanisms. The newly described process involves the transfer of molecules of up to $500 \mathrm{kDa}$ for free percolation and convection in the interstitial fluid outside the reticular conduits; it is perfectly intact in mice after depletion of subcapsular sinus macrophages; it is a receptorindependent, fluid-phase process; and it takes place through sinusoidal/subcapsular sinus LECs (Supplemental Figure 8). The entry of s.c. administered antibodies into the draining LN was observed earlier in a few experiments using sophisticated immunization protocols and adoptive transfer of lymphocytes, high doses $(20-25 \mu \mathrm{g})$ 

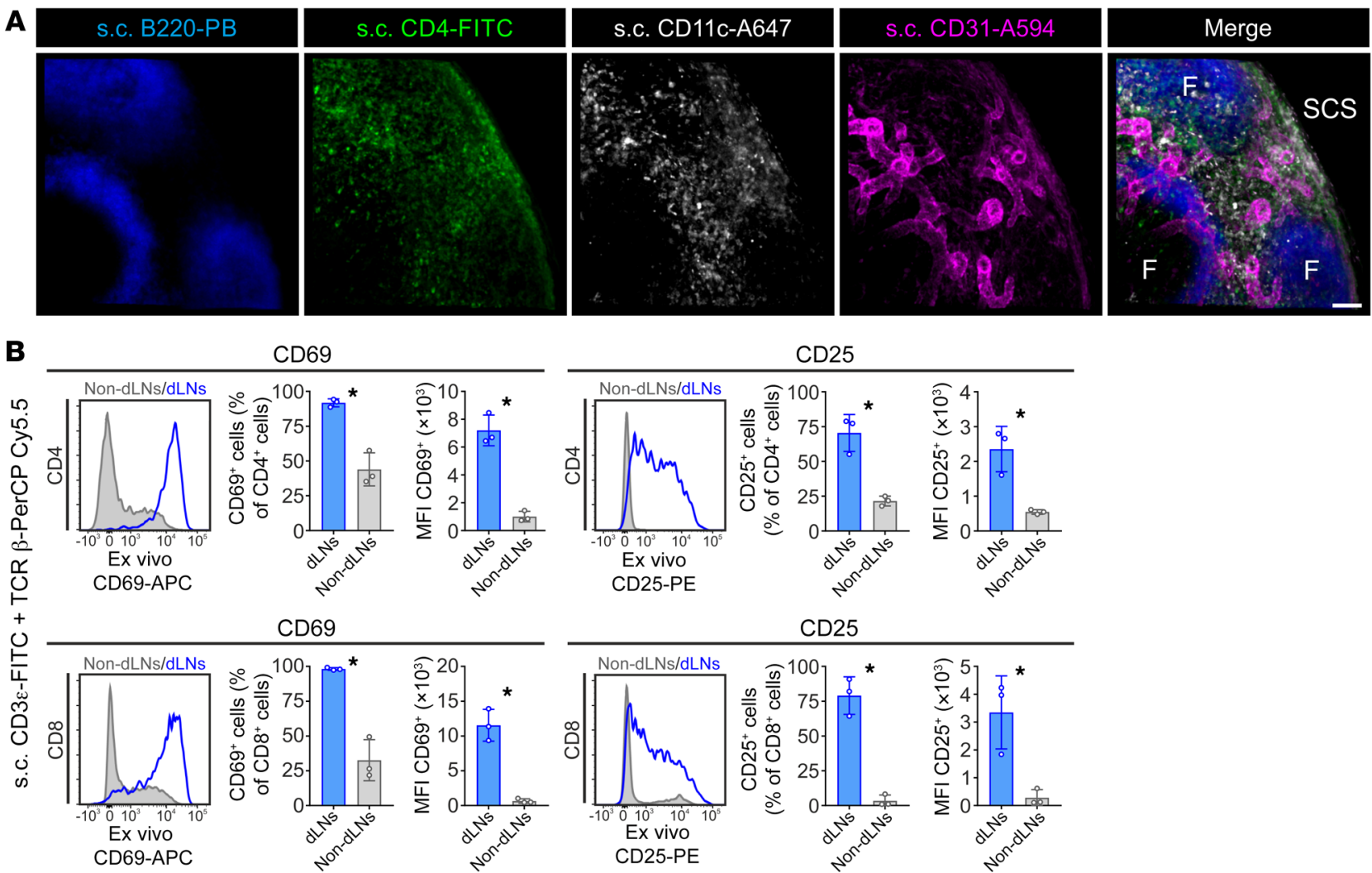

CD69

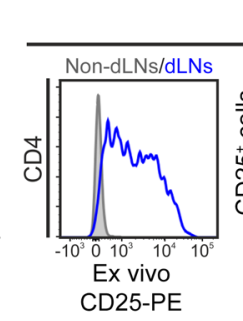

CD25
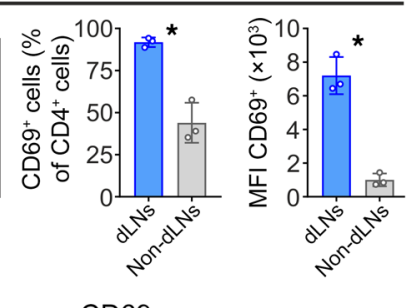

CD69
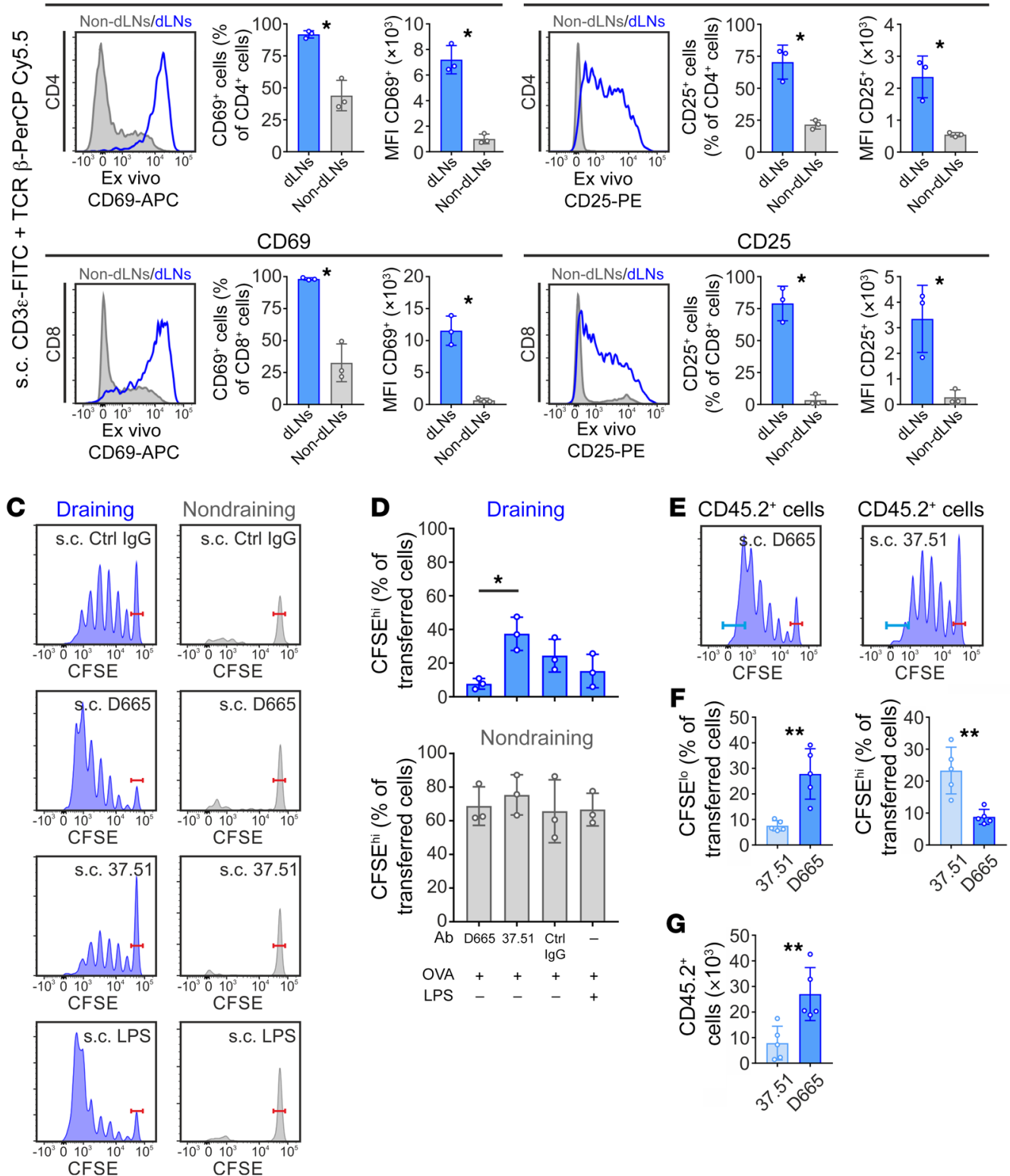

Figure 11. Antibody transcytosis allows efficient whole-mount imaging and spatially controlled lymphocyte activation in the draining LNs. (A) Wholemount imaging of an optically cleared draining LN after s.c. injection of the indicated fluorochrome-conjugated antibodies (10 $\mu \mathrm{g}$ each, $t=30 \mathrm{~min}, n=4)$. Scale bar: $50 \mu \mathrm{m}$. (B) Flow cytometric analyses of lymphocyte activation in draining LNs (dLNs) and nondraining LNs (Non-dLNs) after s.c. administration of anti-CD3 and anti-TCR- $\beta$ antibodies ( $2 \mu \mathrm{g}$ each, $t=18 \mathrm{~h}, n=3$ ). The cells were stained ex vivo for CD69, CD25, CD4, and CD8. (C and D) Representative CFSE dilution histograms of CD45.2+ OTII donor cells (C) and quantification (D) of OTIl lymphocyte proliferation (undivided CFSE ${ }^{\text {hi }}$ cells) in the draining and nondraining popliteal LNs after s.c. administration of OVA and control (IgG), agonistic (clone D665), and function-blocking (clone 37.51) CD28 antibodies $(n=3)$. LPS was used as a positive control. (E-C) The agonistic and function-blocking CD28 antibodies were administered separately to the 2 different legs of the same animal (the same experimental setup as in $\mathbf{C}$ ), and OTII activation (E and $\mathbf{F})$ and total numbers $(\mathbf{G})$ in the draining $L N$ were measured $(n=5)$. In the bar graphs, each dot represents $1 \mathrm{LN}$, and data are the mean $\pm \mathrm{SD}$. ${ }^{*} P<0.05$ and ${ }^{* *} P<0.01$, by Mann-Whitney $U$ test $(\mathbf{B}, \mathbf{F}$, and $\mathbf{G})$ and Kruskal-Wallis followed by Dunn's test (D). Ctrl, control. 
of anti-follicular DC antibodies, and extensive redistribution durations (24-48 hours), but the pathways involved were not analyzed $(50,51)$. Our current analyses demonstrate the transcytotic antibody transfer to multiple targets on the draining LN using antibody doses that were more than 200-fold-lower and distribution times that were more than 2800-fold shorter under strictly physiological conditions. In conjunction with elegant real-time analyses of conduit filling, it has also been reported that large foreign proteins are found in the subsinusoidal interstitial space of the draining $\mathrm{LN}$ after 6 minutes, but the route or mechanism of the transfer was not elucidated in any way $(10,52)$. Our analyses thus reveal that vesicular fluid-phase transfer through LECs into the subcapsular sinus represents a third major pathway for protein transfer into the stroma of the draining LN (Supplemental Figure 8).

Interestingly, the uptake of s.c. injected antibodies and dextrans of $70 \mathrm{kDa}$ or more in the $\mathrm{LN}$ parenchyma was not reported in the elegant landmark study by Gretz et al., which evaluated lymph filtration by microscopy (7). We speculate that when they visualized the high local concentrations of the labeled molecules in the narrow subcapsular sinus and reticular conduits, dextrans diffusing throughout the parenchymal interstitium may have remained below the detection threshold, as was, in fact, the case in our own previous analyses (11). Moreover, Gretz et al. used a single antiMHC I antibody, a large fraction of which will bind already in the skin and lymphatic vessels to MHC I expressed at high levels on all nucleated cells, which possibly hampered its detectability in the microscopic studies of the LN parenchyma. Using the same anti-MHC I antibody clone, conjugate, and dose, we demonstrate by flow cytometric and microscopic analyses that this antibody is also transferred to the parenchyma of draining LNs (Supplemental Figure 3, A and B). Collectively, our analyses with 2 high-molecular-weight dextrans, a 180-kDa nonantibody protein antigen, and almost 40 different antibodies clearly show the robust transfer of large lymph-borne proteins and polysaccharides through the subcapsular sinus floor to the parenchyma of draining LNs.

The transfer of lymph-borne antibodies to the draining LN is immunologically relevant under both physiological and pathological conditions. IgG is one of the most abundant proteins in the interstitial fluid of healthy skin (concentration of $\sim 5 \mu \mathrm{g} / \mu \mathrm{l}$ ) and in the lymph proteome $(13,14)$. Under steady-state conditions, IgG concentrations in the afferent lymph reach approximately $20 \%$ of those measured in plasma (i.e., lymph concentration of $\sim 5 \mu \mathrm{g} / \mu \mathrm{l}$ ) and are even higher in the efferent lymph also containing the Ig produced locally in the LN (53). In inflammation, the amount of Ig in the interstitial fluid and lymph further increases as a result of the changes in capillary walls, which allow paracellular leakage of plasma Ig into the extravascular tissues and extravasation of Ig-producing plasma cells into sites of inflammation $(13,54$, 55). In addition, very high levels of antibodies are introduced to the skin in clinical settings when therapeutic antibodies such as trastuzumab (anti-HER2), adalimumab (anti-TNF), denosumab (anti-RANKL), and ustekinumab (anti-IL-12 and anti-IL-23) are administered s.c. for systemic delivery. The antibody concentration in these injections is typically approximately $100 \mu \mathrm{g} / \mu \mathrm{l}$. Thus, the transcytosis mechanism identified here will instantly deliver intact endogenous and exogenous monomeric antibodies from the periphery through the subcapsular sinus floor to the parenchy- ma of the draining LN, where they can execute any of their multiple effector and signaling functions.

We discovered that the large lymph-borne molecules traverse the subcapsular sinus lining LECs by fluid-phase transcytosis (Supplemental Figure 8). We observed that the s.c. administered nonbinding antibody transits via intracellular vesicles in LECs using both immunoelectron microscopy and confocal imaging of EEA1 ${ }^{+}$early endosomes. Antibody entry via the paracellular route is unlikely, since we observed no antibody signal at the interendothelial junctions with any of the imaging techniques, and tightening of the endothelial junctions with adrenomedullin did not inhibit the transfer. Different types of endocytotic processes display characteristic ligand dependency, vesicular morphology, and molecular machinery (56-59). The dynamin dependence of the transfer of lymph-borne cargo through sinusoidal LECs would have been compatible with the possibility that either macropinocytosis or caveolae- or clathrin-mediated endocytosis was involved and argued against the role for dynamin-independent forms of the endocytosis. However, we did not observe, either by immunoelectron or confocal microscopy, any formation of macropinocytic vesicles during the transfer, and the process was insensitive to 2 different inhibitors of macropinocytosis. Furthermore, we observed normal antibody transfer in the $\mathrm{Cav1}^{-/-}$mice (32), excluding the role of caveolae-dependent endocytosis and transcytosis in the process. Clathrin-mediated endocytosis, on the other hand, is typically triggered by receptor ligation and only involves small volumes of passive fluid transport, takes place via regularly shaped round vesicles, and is a relatively slow process that requires approximately 2 minutes in every cell type studied (57). Our findings showing that antibody transcytosis is receptor independent, takes place in elongated tubulovesicular organelles, and has extremely fast kinetics (<30 s from the s.c. injection) are not compatible with a clathrin-dependent process. Moreover, 2 structurally distinct clathrin inhibitors did not interfere with the antibody transfer. We therefore believe that the majority of antibody transfer through the sinus floor takes place independently of macropinocytosis and caveolae and clathrin vesicles, although we do not imply that antibodies would be specifically excluded from the fluid volume entering these types of vesicles if their formation is triggered by other ligands. Although the analyses of endocytosis under in vivo settings in LNs remain technically and methodologically challenging, we find it interesting that the transfer process through the sinus floor has apparent similarities to fast and ultrafast forms of endocytosis, which have so far been identified only in synaptic terminals $(56,58,59)$.

The transcytosis pathway identified here can be harnessed for several purposes. We believe it opens new possibilities for bioimaging by allowing very fast in vivo labeling of parenchymal LN cells in situ using pools of s.c. administered fluorochrome-conjugated antibodies. In addition, this pathway allows the precise manipulation of immune responses by antibodies in a given LN in a spatially highly localized manner. Therefore, it could be potentially exploited to develop new, thoroughly characterized antibody-based adjuvants for vaccines. The parenchymal uptake of lymph-borne antibodies could be useful for delivering check-point inhibitors or tumor cell-depleting antibodies to the tumor draining LNs. When compared with systemic treatments, this approach 
would have clear dose-sparing and precision targeting potential, since the lymphatic drainage will carry peri- or intratumorally injected therapeutic antibodies by default to the same LNs, which drain the metastatic cells escaping from the primary tumor.

In conclusion, we have discovered what we believe to be a constitutively active, mechanistically novel mode of lymph filtering by LECs in LNs that allows extremely efficient fluid-phase transcytosis of any large biomolecule $(<500 \mathrm{kDa})$ through the sinus floor to the parenchyma of draining LNs in an intact form within seconds. Targeted delivery of s.c. administered antibodies selectively to the draining $L N$ opens new avenues for harnessing the filtering functions of LNs for immune modulation.

\section{Methods}

Mice. WT C57BL6/J, C57BL6/N, SJL, and BALB/c mice were purchased from Charles River Laboratories and Janvier Labs. CavitmIMls/1 (Cav1 ${ }^{-1}$, stock 004585) (32), $\mathrm{FCRn}^{-/-}$(60), and OTII (expressing transgenic $\alpha \beta$ T cell receptors [TCRs] that are specific for chicken OVA) (61) mice were obtained from The Jackson Laboratory. Six- to tenweek-old mice of both sexes (age- and sex-matched animals within each experiment) were used.

Antibodies. The antibodies used in this study are listed in Supplemental Table 1. $(\mathrm{Fab})_{2}$ fragmentation of MEL-14 and 9-11 antibodies was custom-generated by GenScript. When indicated, antibodies were biotinylated using $\mathrm{N}$-hydroxysuccinimide ester (NHS) biotin, conjugated to HRP using the HRP Conjugation Kit (ab102890, Abcam), or treated with PNGase F using the Rapid PNGase F Digestion Kit (nonreducing format; New England BioLabs).

Subcutaneous administration of antibodies and dextrans. The antibodies were filtered using a $10-\mathrm{kDa}$ cutoff centrifugal filter unit (MRCPRT010, MilliporeSigma) to remove any possible free fluorochromes and, when necessary, to concentrate the antibodies. The recipient mice were slightly anesthetized using isoflurane, and the antibodies were injected s.c. into the dorsal aspect of hind paws in 10 to $20 \mu \mathrm{l}$ volume using Microfine Demi 0.3-ml syringes (BD) and a 30-G needle, unless otherwise indicated. The dose and in vivo distribution time of the s.c. injected antibodies are specified in the text and figure legends.

TRITC-conjugated, lysine-fixable 70-kDa dextran (D1818, Invitrogen, Thermo Fisher Scientific) and FITC-conjugated, lysine-fixable 500-kDa dextran (D7136, Invitrogen, Thermo Fisher Scientific) were injected s.c. $(50 \mu \mathrm{g} / \mathrm{paw})$.

At the end of the experiments, the mice were sacrificed (cervical dislocation for short distribution time points and carbon dioxide asphyxia for longer ones), and the draining LNs (ipsilateral popliteal), the second LN in the chain (lumbar), and nondraining LNs (axillary and contralateral popliteal LNs) were rapidly dissected free (Figure 1A). We verified the lymphatic drainage pathway from the skin by s.c. Evans blue dye injections (data not shown). The time interval between cervical dislocation and freezing of the draining popliteal LN was less than 30 seconds.

Intravenous administration of antibodies. When indicated, Pacific Blue-conjugated anti-B220, FITC-conjugated anti-CD4, Alexa Fluor 647-conjugated anti-CD11c, and Alexa Fluor 594-conjugated anti-CD31 (1- to 50- $\mu \mathrm{g}$ doses) were injected i.v. into the tail vein and allowed to circulate for 10 minutes.

For detection of BECs, Alexa Fluor 594-conjugated anti-CD31 antibody was administered via the s.c. route. After 2 minutes, $30 \mathrm{~g}$
Alexa Fluor 488-conjugated anti-PLVAP antibody was injected i.v. (to stain the luminal surface of $\mathrm{BEC}$ ), and the mice were sacrificed 3 minutes later.

Processing of the LNs for microscopy. The isolated LNs were carefully embedded in a predetermined orientation (to produce vertical sections in relation to hilar blood vessels) in OCT medium under a stereomicroscope. The samples were snap-frozen on dry ice, stored at $-70^{\circ} \mathrm{C}$, and used for the cutting of $6-\mu \mathrm{m}$ cryostat sections. When fluorochrome-conjugated antibodies that bind to mouse antigens had been injected s.c., the sections were overlaid with ProLong Gold mounting medium (Thermo Fisher Scientific) without any ex vivo fixations or ex vivo stainings. When indicated (ex vivo staining), the sections were stained in vitro for additional markers.

When the unlabeled free antibody (rat IgG2a isotope control, $553926, \mathrm{BD})$ was used, an in situ fixation step was included. Thirty seconds after s.c. injections of the free antibody, $4 \%$ paraformaldehyde solution was injected s.c. After 90 seconds, the mice were sacrificed, LNs isolated, and cryosections cut. Before stainings, the sections were further fixed with ice-cold $4 \%$ paraformaldehyde for 5 minutes at $4^{\circ} \mathrm{C}$. The sections were then washed with PBS, quenched with $0.1 \mathrm{M}$ glycine, and washed with PBS. The s.c. injected free antibody was visualized by ex vivo staining of the sections with a species-specific Alexa Fluor 488-conjugated anti-rat IgG antibody. When indicated, other marker antibodies were added to the sections after washings.

The LNs from mice injected s.c. with lysine-fixable dextrans were fixed with paraformaldehyde in situ and ex vivo, as described above. Popliteal LNs from untouched mice were fixed and processed identically to serve as controls.

For EEA1 stainings, the frozen sections were fixed (4\% paraformaldehyde), quenched ( $50 \mathrm{mM} \mathrm{NaH}_{4} \mathrm{Cl}$ ), blocked (30\% horse serum), and permeabilized ( $0.3 \%$ Triton $\mathrm{X}$ ). The sections were incubated with an anti-EEA1 antibody overnight at $+4^{\circ} \mathrm{C}$ followed by an anti-rabbit Alexa Fluor 546 secondary antibody. Finally, CD31 was used to stain for subcapsular sinus LECs, and DAPI was added for nuclear detection.

Confocal microscopy and image analyses. The immunofluorescently stained sections were examined using an LSM 780 confocal microscope (Carl Zeiss) with Plan-Apochromat 20×/0.8 and c-Apochromat Korr M27 40×/1.20 objectives and Zen 2010 software (Carl Zeiss). Pinhole adjustments were used to produce the same slice thickness (typically $1.2-\mu \mathrm{m}$ thickness) for each channel.

Image analyses were performed using ImageJ software (NIH). The background was subtracted from all images (except Figure 8A), and linear brightness adjustments were made in certain cases. Brightness adjustments and thresholds were always applied equally to all images used in comparisons. Quantifications of staining intensities for the free antibody and dextrans in LN zones 1-6 (zone 1 corresponds to the subcapsular sinus and zone 6 to the deep paracortical area) were done by measuring the MFI of regions of interest using ImageJ software. The staining intensities along reference lines across the conduits were measured using the ImageJ plugin RGB Profiler. In these experiments, the core of the conduits was defined by maximal collagen I staining and the outer surfaces of the conduits by the peaks of ER-TR7 staining intensities. For certain representative images, maximal projections were generated from $Z$-stacks, and noise was reduced using the mean filter function in ImageJ.

$\mathrm{EEA1}^{+}$vesicular structures were imaged using an LSM 880 confocal microscope (Carl Zeiss) with an Airyscan detector (Carl Zeiss) and 
a C Plan-Apochromat $63 \times / 1.4$ oil objective. Images were acquired and processed with Zen 2.3 SP1 FP2 software (Carl Zeiss). Images were taken from a single plane, or $Z$-stacks were imaged from the subcapsular sinus with a $0.15-\mu \mathrm{m}$ slice interval from a total thickness of 1.75 to $2.03 \mu \mathrm{m}$. Linear brightness adjustments and image analysis were done using Imaris 8.1.2 software (Bitplane). The "Surface" tool was used to create a mask of CD31+ floor LECs, and the "Spots" tool was used to detect vesicular structures formed by the free antibody $(0.3-\mu \mathrm{m}$ radius; distinguished on the basis of signal intensity over a cutoff value set with the negative control). The surface of EEA1 ${ }^{+}$vesicles was modeled in 3D using the "Surface" tool. The free antibody vesicles and their contact with EEA1 ${ }^{+}$vesicles were enumerated manually. A video portraying the free antibody and EEA1 vesicles was recorded and converted to QuickTime files using Imaris 8.1.2.

A 3i Spinning Disk confocal microscope (Intelligent Imaging Innovations) with an LD c-Apochromat $40 \times / 1.1 \mathrm{~W}$ objective was used to image cross-sectional areas of whole LNs. Images were acquired using SlideBook 6 software (Intelligent Imaging Innovations). Background subtractions, linear brightness adjustments, and mean filter noise reductions were done using ImageJ software.

Optical clearing and whole-mount imaging. For the whole-mount stainings, we injected $10 \mu \mathrm{g}$ fluorochrome-labeled antibodies s.c. and isolated the draining LNs after 30 minutes. The methodology for the optical clearing of whole-mount samples with benzyl alcohol and benzyl benzoate (BABB) was adapted from ref. 62 and performed as described previously $(11,63)$.

The samples were imaged with an LSM 780 confocal microscope (Carl Zeiss) using a Plan-Apochromat 20×/0.8 objective and Zen 2010 software. Subtraction of the background and linear brightness adjustments were performed using Imaris 8.1.2 software. 3D image reconstruction was generated from $Z$-stacks ( $5-\mu \mathrm{m}$ slice thickness) using Imaris 8.1.2 software, and the results were converted to QuickTime files.

Transmission and immunoelectron microscopy. Untouched LNs were collected and processed for transmission electron microscopy as previously described (11).

For immunoelectron microscopy, the free antibody was administered s.c. for 2 minutes. The draining LNs were then perfused with prewarmed $\mathrm{PBS}\left(37^{\circ} \mathrm{C}\right)$, perfusion fixed $(0.01 \mathrm{M}$ periodate, $0.075 \mathrm{M}$ lysine, $0.037 \mathrm{M} \mathrm{Na}$ phosphate buffer with $2 \%$ paraformaldehyde and $0.2 \%$ glutaraldehyde), collected, immersion fixed in the same buffer for 1 hour at room temperature, and cryoprotected with $2.1 \mathrm{M}$ sucrose overnight at $4^{\circ} \mathrm{C}$. The LNs were then extensively washed in a phosphate buffer, snap-frozen, cut into $10-\mu \mathrm{m}$ sections, and stained with $10 \mathrm{~nm}$ gold-conjugated goat anti-rat IgG (EM.GAT10, BBI Solutions) in a phosphate buffer containing $0.5 \%$ fish gelatin and $0.5 \%$ BSA for 3 hours at room temperature. After postfixation, dehydration, and embedment on the microscope slides, polymerized blocks were detached, and 70-nm sections were cut using an ultramicrotome. Finally, the sections were stained with $1 \%$ uranyl acetate and $0.3 \%$ lead citrate and examined using a JEM-1400 Plus transmission electron microscope (JEOL).

Flow cytometry. After collecting the LNs, lymphocytes were mechanically teased from the tissue and filtered. When fluorochrome-conjugated primary antibodies were injected in vivo, the suspended cells were subjected to FACS analyses without any further processing. When unconjugated antibodies were administered, the cells were stained ex vivo with Alexa Fluor 488-conjugated second-stage antibodies. When indicated, fluorochrome-conjugated antibodies against other leukocyte surface markers were added during the ex vivo processing steps. Before ex vivo stainings, the samples were treated with Fc block and stained with Fixable Viability Dye eFluor 780 (eBioscience).

The cells were analyzed using a BD LSRFortessa flow cytometer. For comparisons between different groups, the data were always acquired using identical instrument settings. All flow cytometric data were analyzed using Flow Jo software (TreeStar).

In selected experiments, CD $45.2^{+}$mice were injected s.c. with the antibody pool. After collecting the draining popliteal LN, it was pooled into the same tube with an untouched popliteal LN isolated from an uninjected CD45.1 $1^{+}$mouse. The lymphocyte suspension was then made, and the binding of s.c. administered antibodies on CD $45.1^{+}$and CD $45.2^{+}$cells was separately analyzed by flow cytometry.

Competition experiments. The function of the Fc receptors CD16 and CD32 in the antibody transfer was analyzed by s.c. injection of a function-blocking antibody (2.4G2, Fc block; ref. 16) or vehicle alone. After 5 minutes, a second dose of Fc block (or vehicle alone), together with the directly labeled test antibodies, was administered s.c. to the same paw.

For competition experiments with unlabeled Igs, a pool of fluorochrome-conjugated test antibodies was mixed in vitro with a 100-fold molar excess of unlabeled rat IgG2a monoclonal antibody (anti-CD44 antibody 9B5) or with purified polyclonal mouse Ig, and the mixture was then administered s.c. to mice. Control animals obtained the same pool of fluorochrome-conjugated test antibodies diluted in the vehicle (PBS).

In vivo clodronate depletions. To deplete macrophages from the draining LNs, a single injection of clodronate liposomes (or vehicleloaded liposomes as controls) was administered s.c. into the anterolateral surface of the tibia on day 0 (23). Three days later, the test antibodies were administered s.c. into the footpad of the same paw.

Treatments with endocytosis inhibitors in vivo. The mice were anesthetized and the popliteal LNs were surgically exposed with minimal tissue trauma. Different inhibitors were applied directly to the exposed left popliteal LN, and the exposed right popliteal LN was similarly treated with control solutions with approximately $50-\mu l$ droplets of prewarmed solution. Fresh droplets were instilled at 2- to 3-minute intervals for 15 minutes. Thereafter, a pool of fluorochrome-conjugated antibodies was administered s.c. to both paws and allowed to distribute for 5 minutes before the mouse was sacrificed.

The following endocytosis inhibitors and controls were used: clathrin inhibitor Pitstop 2 (33) (Abcam) and its specific negative control (Pitstop 2 control, Abcam) both at $75 \mu \mathrm{M}$; the clathrin inhibitor monodansylcadaverine (34) (MilliporeSigma; $100 \mu \mathrm{M}$ ) and its DMSO vehicle control; the macropinocytosis inhibitor EIPA (36) [5-( $N$-ethyl$N$-isopropyl amiloride, MilliporeSigma; $100 \mu \mathrm{M}]$ and its DMSO vehicle control; the macropinocytosis inhibitor imipramine (37) (MilliporeSigma, $15 \mu \mathrm{M})$ and its PBS vehicle control; the dynamin inhibitor Dyngo-4a (38) (MilliporeSigma, $400 \mu \mathrm{M}$ ) and its DMSO vehicle control; and the dynamin inhibitor Dynole 34-2 (39 (Abcam) and its specific control Dynole 31-2 (Abcam), both at $75 \mu \mathrm{M}$. In addition, we applied adrenomedullin (Phoenix Pharmaceuticals; $100 \mathrm{nM}$ ), which stabilizes interendothelial junctions (40), and its vehicle control (PBS) using the same protocol.

Antigen uptake by resident antigen-presenting cells. Recombinant human AOC3 protein (180-kDa MW; Peprotech) was conjugated to Alexa Fluor 647 using a Microscale Protein Labeling Kit (Thermo Fisher Scientific). After filtering, $2 \mu \mathrm{g}$ protein was injected s.c. into mice. 
Uninjected mice served as controls in all experiments. After 2 hours, the draining LNs were isolated and used for flow cytometry or snapfrozen for immunohistochemical analysis. For flow cytometric analyses, single-cell suspensions were prepared by digesting minced pieces for 30 minutes at $37^{\circ} \mathrm{C}$ in a buffer (HBSS) containing collagenase D $(1 \mathrm{mg} / \mathrm{ml})$ and DNAse I $(50 \mu \mathrm{g} / \mathrm{ml})$. The cells were then stained ex vivo for CD45, CD3, CD19, CD11b, CD11c, MHC II, F4/80, and CD169 and analyzed using the BD LSRFortessa. For immunohistochemical analyses, frozen sections were cut and stained ex vivo for CD11c.

Local $T$ cell activation by s.c. administered stimulatory antibodies. Anti-TCR (clone H57-597, PerCP Cy5.5-conjugated) and antiCD3 (clone 145-2C11, FITC-conjugated) antibodies (3 $\mu$ g each) were administered s.c. into the left hind paw. Isotype-matched control antibodies were similarly injected into control mice. One group of mice was sacrificed after 5 minutes, and the rest of the mice were sacrificed after 18 hours. The draining and nondraining LNs were isolated and stained ex vivo using fluorochrome-conjugated antibodies against CD4, CD8, and the early T cell activation markers CD69 and CD25.

In another experimental setup, splenocytes were isolated from CD45.2+ OTII donor mice expressing a transgenic TCR for OVA (61). Untouched naive $\mathrm{CD} 4^{+}$lymphocytes were enriched from the splenocytes using a negative MACS Selection Kit (Miltenyi Biotec). The purified cells (typically more than $90 \%$ of L-selectin ${ }^{\text {hi }} \mathrm{CD} 44^{\text {lo }} \mathrm{CD} 4^{+}$cells) were fluorescently labeled with $1 \mu \mathrm{M}$ CFSE for 30 minutes at $37^{\circ} \mathrm{C}$. The labeled cells were then adoptively transferred into CD $45.1^{+}$congenic recipients via i.v. injections $\left(5 \times 10^{6}\right.$ cells/mouse $)$ and allowed to redistribute to LNs and other organs for 18 hours. Thereafter, an agonistic anti-CD28 antibody (clone D665) (47), an antagonistic antiCD28 antibody (clone 37.51), or a control antibody together with 2 $\mu \mathrm{g}$ OVA antigen were administered s.c. to the recipient mice. Unlike what is observed in vitro, the clone 37.51 antibody functions as a CD28 antagonist in vivo $(48,49,64)$. Coinjection of OVA and LPS $(2 \mu \mathrm{g}$, from Escherichia coli O111:B4, MilliporeSigma) as an adjuvant served as a positive control. After 3 days, the draining and nondraining popliteal LNs were isolated. The cells were stained ex vivo for CD45.2, and the dilution of CFSE labels in the transferred cell population was determined using flow cytometry. For quantification of cell division, the percentages of the labeled OTII cells in the CFSE ${ }^{\text {hi }}$ (undivided) and $\mathrm{CFSE}^{\mathrm{lo}}$ (divided $\geq 5$ times) cell populations were determined. In certain experiments, the agonistic CD28 antibody was injected into the left hind paw and the antagonistic CD28 antibody into the right hind paw of the same animal. The absolute numbers of OTII T cells in each LN were counted by determining the absolute numbers of lymphocytes and the percentage of $\mathrm{CFSE}^{+}$(any intensity) cells in the LNs.
Statistics. Littermate WT mice of the same sex were randomly assigned to the experimental groups. When gene-modified mice were used, age-, sex-, and strain-matched WT mice were chosen as controls. The investigators were not blinded to mouse allocation during the experiments or outcome assessments. All data from experiments in which the positive and/or negative controls worked appropriately were included in the study. Numeric data are given as the mean \pm SD. Comparisons between 2 treatment groups or genotypes were performed using the Mann-Whitney $U$ test or the Wilcoxon matched-pairs, signed-rank test. Comparisons between multiple groups were done using the Kruskal-Wallis test followed by Dunn's test when appropriate. Linear regression analyses were used to analyze fluorescence intensities across different zones of the LNs. SAS 9.4 statistical software and GraphPad Prism, version 7 (GraphPad Software) were used for statistical analyses. $P$ values of less than 0.05 were considered statistically significant.

Study approval. The animal experiments were performed in accordance with the 3R-principle and in adherence to the rules and regulations of the Finnish Act on Animal Experimentation (497/2013). They were approved by the Ethics Committee for Animal Experimentation in Finland (animal license numbers 5587/04.10.07/2014 and 6211/04.10.07/2017).

\section{Author contributions}

LK, RFM, and KA contributed to the study design, performed experiments, and analyzed data. PR provided expertise on in vivo experimentation, and SJ provided resources for the research. JI planned and performed endocytosis and transcytosis experiments. MS conceived and supervised the study, planned the experiments, analyzed data, and wrote the manuscript. All authors commented on the manuscript.

\section{Acknowledgments}

We thank E.L. Väänänen and R. Sjöroos from MediCity, University of Turku, for their expert technical assistance. We acknowledge the members of the Cell Imaging Core at the Turku Centre for Biotechnology and the Laboratory of Electron Microscopy at the University of Turku. This research was supported by grants from the Academy of Finland (312517, to JI; 303802, to SJ; and 276401 and 315947 , to MS) and the Juselius Foundation (16032016, to MS).

Address correspondence to: Marko Salmi, MediCity Research Laboratory, University of Turku, Tykistökatu 6 A, FIN-20520 Turku, Finland. Phone: 358.50.3385678; Email: marko.salmi@utu.fi.
1. Girard JP, Moussion C, Förster R. HEVs, lymphatics and homeostatic immune cell trafficking in lymph nodes. Nat Rev Immunol. 2012;12(11):762-773.

2. Randolph GJ, Ivanov S, Zinselmeyer BH, Scallan JP. The lymphatic system: integral roles in immunity. Annu Rev Immunol. 2017;35:31-52.

3. Petrova TV, Koh GY. Organ-specific lymphatic vasculature: from development to pathophysiology. JExp Med. 2018;215(1):35-49.

4. Wiig H, Swartz MA. Interstitial fluid and lymph formation and transport: physiological regulation and roles in inflammation and cancer. Physiol Rev. 2012;92(3):1005-1060.
5. Baluk P, et al. Functionally specialized junctions between endothelial cells of lymphatic vessels. JExp Med. 2007;204(10):2349-2362.

6. Gray EE, Cyster JG. Lymph node macrophages. J Innate Immun. 2012;4(5-6):424-436.

7. Gretz JE, Norbury CC, Anderson AO, Proudfoot AE, Shaw S. Lymph-borne chemokines and other low molecular weight molecules reach high endothelial venules via specialized conduits while a functional barrier limits access to the lymphocyte microenvironments in lymph node cortex. J Exp Med. 2000;192(10):1425-1440.

8. Palframan RT, et al. Inflammatory chemokine transport and presentation in HEV: a remote control mechanism for monocyte recruitment to lymph nodes in inflamed tissues. J Exp Med. 2001;194(9):1361-1373.

9. Sixt M, et al. The conduit system transports soluble antigens from the afferent lymph to resident dendritic cells in the T cell area of the lymph node. Immunity. 2005;22(1):19-29.

10. Roozendaal R, et al. Conduits mediate transport of low-molecular-weight antigen to lymph node follicles. Immunity. 2009;30(2):264-276.

11. Rantakari $\mathrm{P}$, et al. The endothelial protein PLVAP in lymphatics controls the entry of lymphocytes 
and antigens into lymph nodes. Nat Immunol. 2015;16(4):386-396.

12. Malhotra D, et al. Transcriptional profiling of stroma from inflamed and resting lymph nodes defines immunological hallmarks. Nat Immunol. 2012;13(5):499-510.

13. Worm AM. Exchange of macromolecules between plasma and skin interstitium in extensive skin disease. J Invest Dermatol. 1981;76(6):489-492.

14. Leak LV, et al. Proteomic analysis of lymph. Proteomics. 2004;4(3):753-765.

15. Pyzik M, Rath T, Lencer WI, Baker K, Blumberg RS. FcRn: the architect behind the immune and nonimmune functions of IgG and albumin. J Immunol. 2015;194(10):4595-4603.

16. Unkeless JC. Characterization of a monoclonal antibody directed against mouse macrophage and lymphocyte Fc receptors. J Exp Med. 1979;150(3):580-596.

17. Bournazos S, Wang TT, Dahan R, Maamary J, Ravetch JV. Signaling by antibodies: recent progress. Annu Rev Immunol. 2017;35:285-311.

18. Lee W, Syed Atif A, Tan SC, Leow CH. Insights into the chicken IgY with emphasis on the generation and applications of chicken recombinant monoclonal antibodies. J Immunol Methods. 2017;447:71-85.

19. Kaldjian EP, Gretz JE, Anderson AO, Shi Y, Shaw S. Spatial and molecular organization of lymph node $\mathrm{T}$ cell cortex: a labyrinthine cavity bounded by an epithelium-like monolayer of fibroblastic reticular cells anchored to basement membrane-like extracellular matrix. Int Immunol. 2001;13(10):1243-1253.

20. Katakai T, Hara T, Sugai M, Gonda H, Shimizu A. Lymph node fibroblastic reticular cells construct the stromal reticulum via contact with lymphocytes. JExp Med. 2004;200(6):783-795.

21. Szakal AK, Holmes KL, Tew JG. Transport of immune complexes from the subcapsular sinus to lymph node follicles on the surface of nonphagocytic cells, including cells with dendritic morphology. J Immunol. 1983;131(4):1714-1727.

22. Phan TG, Grigorova I, Okada T, Cyster JG. Subcapsular encounter and complement-dependent transport of immune complexes by lymph node B cells. Nat Immunol. 2007;8(9):992-1000.

23. Iannacone M, et al. Subcapsular sinus macrophages prevent CNS invasion on peripheral infection with a neurotropic virus. Nature. 2010;465(7301):1079-1083.

24. Fletcher AL, Acton SE, Knoblich K. Lymph node fibroblastic reticular cells in health and disease. Nat Rev Immunol. 2015;15(6):350-361.

25. Fung KYY, Fairn GD, Lee WL. Transcellular vesicular transport in epithelial and endothelial cells: challenges and opportunities. Traffic. 2018;19(1):5-18.

26. Vasile E, Simionescu M, Simionescu N. Visualization of the binding, endocytosis, and transcytosis of low-density lipoprotein in the arterial endothelium in situ. JCell Biol. 1983;96(6):1677-1689.

27. Ghinea N, Mai TV, Groyer-Picard MT, Milgrom E. How protein hormones reach their target cells. Receptor-mediated transcytosis of hCG through endothelial cells. J Cell Biol. 1994;125(1):87-97.

28. King GL, Johnson SM. Receptor-mediated transport of insulin across endothelial cells. Science.
1985;227(4694):1583-1586.

29. Pruenster M, et al. The Duffy antigen receptor for chemokines transports chemokines and supports their promigratory activity. Nat Immunol. 2009;10(1):101-108.

30. Lim HY, et al. Lymphatic vessels are essential for the removal of cholesterol from peripheral tissues by SR-BI-mediated transport of HDL. Cell Metab. 2013;17(5):671-684.

31. Mills IG, Jones AT, Clague MJ. Involvement of the endosomal autoantigen EEA1 in homotypic fusion of early endosomes. Curr Biol. 1998;8(15):881-884

32. Razani B, et al. Caveolin-1 null mice are viable but show evidence of hyperproliferative and vascular abnormalities. J Biol Chem. 2001;276(41):38121-38138.

33. von Kleist L, et al. Role of the clathrin terminal domain in regulating coated pit dynamics revealed by small molecule inhibition. Cell. 2011;146(3):471-484.

34. Schlegel R, Dickson RB, Willingham MC, Pastan IH. Amantadine and dansylcadaverine inhibit vesicular stomatitis virus uptake and receptormediated endocytosis of alpha 2-macroglobulin. Proc Natl Acad Sci U S A. 1982;79(7):2291-2295.

35. Marques PE, Grinstein S, Freeman SA. SnapShot: macropinocytosis. Cell. 2017;169(4):766-766.e1.

36. Koivusalo M, et al. Amiloride inhibits macropinocytosis by lowering submembranous $\mathrm{pH}$ and preventing Rac1 and Cdc42 signaling. J Cell Biol. 2010;188(4):547-563.

37. Lin HP, et al. Identification of novel macropinocytosis inhibitors using a rational screen of Food and Drug Administration-approved drugs. $\mathrm{Br} \mathrm{J}$ Pharmacol. 2018;175(18):3640-3655.

38. McCluskey A, et al. Building a better dynasore: the dyngo compounds potently inhibit dynamin and endocytosis. Traffic. 2013;14(12):1272-1289.

39. Hill TA, et al. Inhibition of dynamin mediated endocytosis by the dynoles--synthesis and functional activity of a family of indoles. JMed Chem. 2009;52(12):3762-3773.

40. Xu W, Wittchen ES, Hoopes SL, Stefanini L, Burridge K, Caron KM. Small GTPase Rap1A/B is required for lymphatic development and adrenomedullin-induced stabilization of lymphatic endothelial junctions. Arterioscler Thromb Vasc Biol. 2018;38(10):2410-2422.

41. Idoyaga J, et al. Specialized role of migratory dendritic cells in peripheral tolerance induction. J Clin Invest. 2013;123(2):844-854.

42. Gerner MY, Kastenmuller W, Ifrim I, Kabat J, Germain RN. Histo-cytometry: a method for highly multiplex quantitative tissue imaging analysis applied to dendritic cell subset microanatomy in lymph nodes. Immunity. 2012;37(2):364-376.

43. Renier N, Wu Z, Simon DJ, Yang J, Ariel P, TessierLavigne M. iDISCO: a simple, rapid method to immunolabel large tissue samples for volume imaging. Cell. 2014;159(4):896-910.

44. Chung K, et al. Structural and molecular interrogation of intact biological systems. Nature. 2013;497(7449):332-337.

45. Tainaka K, et al. Whole-body imaging with singlecell resolution by tissue decolorization. Cell. 2014;159(4):911-924.

46. Yang B, et al. Single-cell phenotyping within transparent intact tissue through whole-body clearing. Cell. 2014;158(4):945-958.

47. Dennehy KM, et al. Cutting edge: monovalency of CD28 maintains the antigen dependence of T cell costimulatory responses. J Immunol. 2006;176(10):5725-5729.

48. Perez VL, Van Parijs L, Biuckians A, Zheng XX, Strom TB, Abbas AK. Induction of peripheral T cell tolerance in vivo requires CTLA-4 engagement. Immunity. 1997;6(4):411-417.

49. Krummel MF, Sullivan TJ, Allison JP. Superantigen responses and co-stimulation: CD28 and CTLA-4 have opposing effects on T cell expansion in vitro and in vivo. Int Immunol. 1996;8(4):519-523.

50. Schwickert TA, et al. In vivo imaging of germinal centres reveals a dynamic open structure. Nature. 2007;446(7131):83-87.

51. Hauser AE, et al. Definition of germinal-center $\mathrm{B}$ cell migration in vivo reveals predominant intrazonal circulation patterns. Immunity. 2007;26(5):655-667.

52. Gerner MY, Casey KA, Kastenmuller W, Germain RN. Dendritic cell and antigen dispersal landscapes regulate T cell immunity. J Exp Med. 2017;214(10):3105-3122.

53. Beh KJ, Watson DL, Lascelles AK. Concentrations of immunoglobulins and albumin in lymph collected from various regions of the body of the sheep. Aust JExp Biol Med Sci. 1974;52(1):81-86.

54. Komarova Y, Malik AB. Regulation of endothelial permeability via paracellular and transcellular transport pathways. Annu Rev Physiol. 2010;72:463-493.

55. Nourshargh S, Alon R. Leukocyte migration into inflamed tissues. Immunity. 2014;41(5):694-707.

56. Watanabe S, Boucrot E. Fast and ultrafast endocytosis. Curr Opin Cell Biol. 2017;47:64-71.

57. Kaksonen M, Roux A. Mechanisms of clathrin-mediated endocytosis. Nat Rev Mol Cell Biol. 2018;19(5):313-326.

58. Ferreira APA, Boucrot E. Mechanisms of carrier formation during clathrin-independent endocytosis. Trends Cell Biol. 2018;28(3):188-200.

59. Cheng JPX, Nichols BJ. Caveolae: one function or many? Trends Cell Biol. 2016;26(3):177-189.

60. Roopenian DC, et al. The MHC class I-like IgG receptor controls perinatal IgG transport, IgG homeostasis, and fate of IgG-Fc-coupled drugs. J Immunol. 2003;170(7):3528-3533.

61. Barnden MJ, Allison J, Heath WR, Carbone FR. Defective TCR expression in transgenic mice constructed using cDNA-based alpha- and beta-chain genes under the control of heterologous regulatory elements. Immunol Cell Biol. 1998;76(1):34-40.

62. Yokomizo T, Yamada-Inagawa T, Yzaguirre AD, Chen MJ, Speck NA, Dzierzak E. Whole-mount three-dimensional imaging of internally localized immunostained cells within mouse embryos. Nat Protoc. 2012;7(3):421-431.

63. Rantakari P, et al. Fetal liver endothelium regulates the seeding of tissue-resident macrophages. Nature. 2016;538(7625):392-396.

64. Yu XZ, Bidwell SJ, Martin PJ, Anasetti C. CD28-specific antibody prevents graftversus-host disease in mice. JImmunol. 2000;164(9):4564-4568. 